\title{
Recursive contracts, lotteries and weakly concave Pareto sets
}

\author{
Harold Cole $^{\mathrm{a}, *}$, Felix Kubler ${ }^{\mathrm{b}}$ \\ a University of Pennsylvania, Philadelphia, PA, United States \\ ${ }^{\mathrm{b}}$ University of Zurich, Zurich, Switzerland
}

\section{A R T I C L E I N F O}

\section{Article history:}

Received 5 November 2011

Revised 26 April 2012

Available online $\mathrm{xxxx}$

\section{JEL classification:}

C61

C63

Keywords:

Recursive contracting

Recursive multipliers

Lotteries

\begin{abstract}
A B S T R A C T
Marcet and Marimon (1994, revised 1998, revised 2011) developed a recursive saddle point method which can be used to solve dynamic contracting problems that include participation, enforcement and incentive constraints. Their method uses a recursive multiplier to capture implicit prior promises to the agent(s) that were made in order to satisfy earlier instances of these constraints. As a result, their method relies on the invertibility of the derivative of the Pareto frontier and cannot be applied to problems for which this frontier is not strictly concave. In this paper we show how one can extend their method to a weakly concave Pareto frontier by expanding the state space to include the realizations of an end of period lottery over the extreme points of a flat region of the Pareto frontier. With this expansion the basic insight of Marcet and Marimon goes through - one can make the problem recursive in the Lagrangian multiplier which yields significant computational advantages over the conventional approach of using utility as the state variable. The case of a weakly concave Pareto frontier arises naturally in applications where the principal's choice set is not convex but where randomization is possible.
\end{abstract}

(c) 2012 Elsevier Inc. All rights reserved.

\section{Introduction}

Marcet and Marimon (1994, revised 1998, revised 2011), in their still unpublished research memo, developed a recursive saddle point method which can be used to solve dynamic contracting problems that include participation, enforcement and incentive constraints. Their method uses a recursive multiplier to capture implicit prior promises to the agent(s) that were made in order to satisfy earlier (in time) instances of these constraints. Prior to their work, the standard method to recursively solve dynamic contracting problems treated the promised utility of the agent(s) as a state variable, and used this state variable to capture the implicit prior promises. The main advantage of the recursive multiplier is that it allows the conditional date $t$ problem to be solved without reference to the ex ante date $t$ payoff to the agent(s). In contrast, when one uses ex ante utility as a state variable, there is an overall ex ante condition as to the allocation of utility across states in $t$ that has to hold, and as a result the date $t$ actions and date $t+1$ continuation utilities must be simultaneously solved as a block. ${ }^{1}$

This computational advantage has led to the recursive multiplier approach being widely used; to cite a few examples Marcet and Marimon (1992), Khan et al. (2003), Cooley et al. (2004), Attanasio and Rios-Rull (2000), Kehoe and Perri (2002), Aiyagari et al. (2002), Atkeson and Cole (2005), Chien et al. (2011). Despite these applications, there remain fundamental

\footnotetext{
We thank seminar participants at the 2011 SED in Ghent as well as an anonymous referee and the editor for helpful comments. We are very grateful to Chris Sleet for pointing out a mistake in an earlier version.

* Corresponding author.

E-mail address: colehl@econ.sas.edu (H. Cole).

1 Messner et al. (2011) provide an extensive discussion of the various ways in which one can construct the dual problems to recursive incentive problems and the conditions under which these methods are valid.
} 
issues with respect to the applicability of these methods. Messner and Pavoni (2004) use a simple example to show that if the Pareto frontier is not strictly concave, then these methods can yield policy functions which are not only suboptimal but infeasible. This is because Marcet and Marimon's method relies on the invertibility of the derivative of the Pareto frontier in order to map the recursive multiplier into the promised utility level. ${ }^{2}$ The method must fail in the presence of flat spots on the Pareto frontier. Public randomization, which is commonly used in environments with nonconvex constraint sets, naturally generates these flat spots on the Pareto frontier. ${ }^{3}$

In this paper we show how one can deal with a weakly concave Pareto frontier by expanding the state space to include the realizations of an end of period lottery over the extreme points on the Pareto frontier that share a common slope. The basic idea is as follows. It can be shown that the value of the recursive programming problem in Marcet and Marimon is the same as the optimal value of the contracting problem even if there are flat spots on the frontier. The problem is that if for a given value of the multiplier the frontier is flat, the recursive multiplier approach yields a continuum of current actions which solve the Bellman equation. It is then impossible to pick the correct action that is consistent with previous periods' incentive constraints. Our approach identifies those actions in these sets that yield extreme payoffs. In the case of one agent and one principal these are the action that yields the highest payoff to the agent and the action that yields the highest payoff to the principal, i.e. the two extreme points of the flat spot of the Pareto frontier. We assume that the principal has access to a public randomization device and can pick a lottery over these two extreme points that satisfies last period's incentive constraints. Under this method, in any period the optimal action is pinned down either by the incentive constraint if it binds, or by the prior selection of the best or worst continuation for the agent (from the last time the incentive constraint did bind) if it does not bind. After we introduce our general method we present an extended example that illustrates our method. A reader less interested in the technical details might want to go to this example in Section 5 first.

The rest of the paper is organized as follows. In Section 2 we describe the general contracting problem. In Section 3 we explain the recursive multiplier approach from Marcet and Marimon and give a simple example to illustrate that without strict concavity this approach cannot yield the correct policy. In Section 4 we describe our solution to the problem and link it to the promised utility approach. Section 5 presents a series of simple examples which clarify our method. In Section 6 we argue that more complicated problems can be solved numerically with our method.

\section{The general problem}

Assume the exogenous shock $\left(s_{t}\right)$ follows a finite Markov chain with transition $\Pi$ and support $\mathcal{S}=\{1, \ldots, S\}$. Each period the principal randomizes over a compact action set $\mathcal{A} \subset \mathbb{R}^{n}$ - to simplify the notation and in anticipation of our results below we assume without loss of generality that he chooses a simple probability distribution (i.e. a distribution with finite support) $\psi_{t}: \mathcal{A} \rightarrow \mathbb{R}_{+}, \psi_{t} \in \Psi$. The choice can depend on the history of realized shocks and realized actions, i.e. $\psi_{t}=\psi\left(h^{t-1}, s_{t}\right)$ where $h^{t-1}=\left(s_{0}, a_{0}, \ldots, s_{t-1}, a_{t-1}\right)$. In a slight abuse of notation we write $\Psi^{\infty}$ to denote the space of all (history-dependent) sequences. We denote the support of $\psi_{t}$ by $\operatorname{supp}\left(\psi_{t}\right)=\left\{a \in \mathcal{A}: \psi_{t}(a)>0\right\}$. Note that the space of all simple probability distributions forms a vector space and convexity is well defined.

It is useful to denote by $\Pi_{t}^{\psi}\left(h^{t+n}\right)$ the conditional probability of history $h^{t+n}$, given history $h^{t}$ and a choice $\left(\psi_{t}\right) \in$ $\Psi^{\infty}$. We assume that there is a fixed initial probability distribution over the shock in period $0, s_{0}$ and $E_{-1}$ denotes the expectation under this distribution. We write the expectation of a function $f($.$) that depends on the realized a_{t+n}$ as

$$
E_{t}^{\psi}\left(f\left(a_{t+n}\right)\right)=\sum_{h^{t+n} \in \operatorname{supp}\left(\Pi_{t}^{\psi}\right)} \Pi_{t}^{\Psi}\left(h^{t+n}\right) f\left(a_{t+n}(h)\right)
$$

We use $E_{t-1, s_{t}}$ for the expectation conditional on a history $h^{t-1}$ and a realized shock $s_{t}-E_{s_{0}}$ denotes the expectation conditional on a realized first period shock, $s_{0}$.

The physical state, $x$, realizes in a compact subset of Euclidean space $\mathcal{X} \subset \mathbb{R}^{m}$ and depends on last period's realized action, last period's state and last period's realized shock but not on the current shock (for our approach, it is trivial to also let the state depend on the current shock, but this adds little to the economics of the model), i.e. the law of motion for $x_{t}$ is given by $x_{t+1}=\zeta\left(x_{t}, a_{t}, s_{t}\right)$.

There are $I$ agents, $i=1, \ldots, I$. The principal can randomize over the actions, but for each agent $i=1, \ldots, I$ the incentive constraint $\left(I C_{i}\right)$ is assumed to hold conditional on each realized action. The participation constraint $\left(P C_{i}\right)$, on the other hand, only has to hold ex ante, before either shock or actions are realized.

\footnotetext{
2 Marcet and Marimon's (2011) revised version of their paper restricts attention to cases in which the Pareto frontier is strictly concave and hence this criticism does not apply.

3 Lotteries naturally arise in environments with incentive constraints, see for example Prescott and Townsend (1984a, 1984b), or discrete choices, see for example Rogerson (1988), and Cole and Prescott (1997).
} 
Our contracting problem then can be written in sequence form as

$$
\max _{\left(\psi_{t}\right) \in \Psi^{\infty}} E_{-1}^{\psi} \sum_{t=0}^{\infty} \beta^{t} r\left(x_{t}, a_{t}, s_{t}\right)
$$

subject to

$$
\begin{aligned}
& x_{t+1}=\zeta\left(x_{t}, a_{t}, s_{t}\right), \\
& p\left(x_{t}, a_{t}, s_{t}\right) \geqslant 0, \quad \forall a_{t} \in \operatorname{supp}\left(\psi_{t}\right), \\
& g^{i}\left(x_{t}, a_{t}, s_{t}\right)+E_{t}^{\psi} \sum_{n=1}^{\infty} \beta^{n} g^{i}\left(x_{t+n}, a_{t+n}, s_{t+n}\right) \geqslant \bar{g}_{1}^{i}, \quad \forall i=1, \ldots, I, \forall a_{t} \in \operatorname{supp}\left(\psi_{t}\right), \\
& E_{-1}^{\psi} \sum_{t=0}^{\infty} \beta^{t} g^{i}\left(x_{t}, a_{t}, s_{t}\right) \geqslant \bar{g}_{2}^{i}, \quad \forall i=1, \ldots, I, \\
& x_{0} \text { given. }
\end{aligned}
$$

For consistency, we assume that for all $(x, a, s) \in \mathcal{X} \times \mathcal{A} \times \mathcal{S}, \zeta(x, a, s) \in \mathcal{X}$ whenever $p(x, a, s) \geqslant 0$. It is useful to combine constraints (2) and (3) - for a fixed $x_{0}$, we write $x_{t}=x\left(h^{t-1}\right)$ to denote the state at $t$ resulting from history $h^{t-1}$ as well as $\mathcal{A}\left(h^{t-1}, s_{t}\right)$ to be the set of all actions satisfying (3) given $x\left(h^{t-1}\right)$ and the current shock $s_{t}$. Since the space of exogenous shocks is assumed to be finite, we frequently write $r_{s}(x, a)$ instead of $r(x, a, s)$ and $g_{s}^{i}(x, a)$ instead of $g^{i}(x, a, s)$. To simplify notation we sometimes use $g^{0}$ to denote the reward function of the principal, i.e. $g^{0}(x, a, s) \equiv r(x, a, s)$. In general we could let the outside option in the (IC) constraint, $\bar{g}_{1}^{i}$ depend on the shock $s$. It will be easy to see that this does not change our analysis, we just drop the dependence to simplify notation.

We make the following assumptions on the fundamentals.

\section{Assumption 1.}

1. The reward functions $r_{s}: \mathcal{X} \times \mathcal{A} \rightarrow \mathbb{R}$ and the constraint functions $g_{s}^{i}: \mathcal{X} \times \mathcal{A} \rightarrow \mathbb{R}$ are continuous and bounded (both from below and above) for all shocks $s \in \mathcal{S}$.

2. There is discounting, i.e. $\beta \in(0,1)$.

3. There is an $\epsilon>0$ such that for each initial condition $\left(x_{0}\right) \in \mathcal{X}$ there exists an action $\left(\psi_{t}\right)$ such that for all agents $i=1, \ldots, I$,

$$
\inf _{h^{t-1}, s_{t}, a_{t} \in \operatorname{supp}\left(\psi_{t}\right)}\left[g^{i}\left(x_{t}, a_{t}, s_{t}\right)+E_{t}^{\psi} \sum_{n=1}^{\infty} \beta^{n} g^{i}\left(x_{t+n}, a_{t+n}, s_{t+n}\right)-\bar{g}_{1}^{i}\right]>\epsilon
$$

and

$$
E_{-1}^{\psi} \sum_{t=0}^{\infty} \beta^{t} g^{i}\left(x_{t}, a_{t}, s_{t}\right)-\bar{g}_{2}^{i}>\epsilon
$$

Assumption 1.1 is perhaps stronger than needed. ${ }^{4}$ Assumption 1.2 and the Slater condition 1.3 are standard. Assumption 1.3 can of course be rewritten in terms of non-randomized actions.

\section{The social planning problem}

In this section, we disregard the participation constraint (5) and focus on a social planner's problem for given welfare weights. Without the participation constraint the planner's problem can be divided into $S$ subproblems, one for each initial shock. To solve the original problem the welfare weight $\gamma$ needs to be chosen to ensure that this constraint holds, i.e. becomes the initial multiplier on the participation constraint.

\subsection{Non-recursive formulation}

For a given choice of $\left(\gamma_{1}, \ldots, \gamma_{I}\right) \in \Gamma=[0, \infty)^{I}$, for a given $s_{0}$ and for an admissible state $x_{0} \in \mathcal{X}$, define the social planner's problem (in sequence form) as

\footnotetext{
4 See Messner et al. (2011) for an example of how weaker assumptions can be shown to be sufficient.
} 


$$
\max _{\left(\psi_{t}\right) \in \Psi^{\infty}} E_{s_{0}}^{\psi}\left[\sum_{t=0}^{\infty} \beta^{t}\left(r\left(x_{t}, a_{t}, s_{t}\right)+\sum_{i=1}^{I} \gamma_{i} g^{i}\left(x_{t}, a_{t}, s_{t}\right)\right)\right]
$$

subject to

$$
\begin{aligned}
& \operatorname{supp}\left(\psi_{t}\right) \subset \mathcal{A}\left(h^{t-1}, s_{t}\right) \quad \text { for all } h^{t-1}, s_{t}, \\
& g^{i}\left(x_{t}, a_{t}, s_{t}\right)+E_{t}^{\psi} \sum_{n=1}^{\infty} \beta^{n} g^{i}\left(x_{t+n}, a_{t+n}, s_{t+n}\right) \geqslant \bar{g}_{1}^{i} \quad \forall a_{t} \in \operatorname{supp}\left(\psi_{t}\right), \forall t, \forall i .
\end{aligned}
$$

\subsubsection{The Lagrangian}

It is useful to introduce the Lagrangian for this problem and to write

$$
\begin{aligned}
L\left(\left(\lambda_{t}^{i}\right),\left(\psi_{t}\right) ;\left(\gamma_{i}\right), x_{0}, s_{0}\right)= & E_{s_{0}}^{\psi} \sum_{t=0}^{\infty} \beta^{t} \\
& \times\left(r\left(x_{t}, a_{t}, s_{t}\right)+\sum_{i=1}^{I}\left(\gamma_{i} g^{i}\left(x_{t}, a_{t}, s_{t}\right)+\lambda_{t, a_{t}}^{i}\left(\sum_{n=0}^{\infty} \beta^{n} g\left(x_{t+n}, a_{t+n}, s_{t+n}\right)-\bar{g}_{1}^{i}\right)\right)\right) .
\end{aligned}
$$

Note that for given $\left(\lambda_{t}\right)$ this function is concave in $\psi$ and the constraint set $\left\{\left(\psi_{t}\right): \operatorname{supp}\left(\psi_{t}\right) \subset \mathcal{A}\left(h^{t-1}, s_{t}\right)\right.$ for all $\left.h^{t-1}, s_{t}\right\}$ is a convex set. Therefore, it is standard to show (e.g. Luenberger, 1969, Theorem 2, p. 221) that if there exist $\left(\psi_{t}\right)^{*}$ with $\operatorname{supp}\left(\psi_{t}^{*}\right) \subset \mathcal{A}\left(h^{t-1}, s_{t}\right)$ for all $h^{t-1}, s_{t}$ and a $\left(\lambda_{t}\right)^{*} \geqslant 0$ that satisfy

$$
L\left(\left(\lambda_{t}\right),\left(\psi_{t}\right)^{*}\right) \geqslant L\left(\left(\lambda_{t}\right)^{*},\left(\psi_{t}\right)^{*}\right) \geqslant L\left(\left(\lambda_{t}\right)^{*},\left(\psi_{t}\right)\right)
$$

for all $\left(\lambda_{t}\right) \geqslant 0$ and all $\left(\psi_{t}\right)$ with $\operatorname{supp}\left(\psi_{t}\right) \subset \mathcal{A}\left(h^{t-1}, s_{t}\right)$ for all $h^{t-1}, s_{t}$ then $\left(\psi_{t}\right)^{*}$ is a solution to the original problem (7).

\subsubsection{A simplifying assumption}

The results in Rustichini (1998) and Dechert (1982) imply that under our assumptions (1)-(3) there exists such a saddle point with the sequences $\left(\lambda_{t}\right)^{*}$ being bounded. However, the analysis is simplified considerably if one can ensure that the sequences are summable.

One easy way to obtain this result is to make the following assumption.

Assumption 2. There exist (sufficiently large) $\bar{\gamma}_{i}$ for all $i=1, \ldots$, I such that in the maximization problem (7), for all $x_{0} \in \mathcal{X}, s_{0} \in \mathcal{S}$ the constraints

$$
g^{i}\left(x_{0}, a_{0}, s_{0}\right)+E_{0}^{\psi} \sum_{n=1}^{\infty} \beta^{n} g^{i}\left(x_{n}, a_{n}, s_{n}\right) \geqslant \bar{g}_{1}^{i} \quad \forall a_{0} \in \operatorname{supp}\left(\psi_{0}\right)
$$

are not binding whenever $\gamma_{i}=\bar{\gamma}_{i}$ and $\gamma \leqslant \bar{\gamma}$. Moreover, whenever $\gamma_{i}=\bar{\gamma}_{i}$ and $\gamma \leqslant \bar{\gamma}$ the optimal solution satisfies the participation constraint (5) for agent $i$.

The assumption is obviously restrictive and it is not necessary for the analysis. However, it simplifies the proof of Theorem 1 below considerably and there are a variety of economic problems where it holds naturally.

A similar theorem is already proven in Marcet and Marimon (1994) without bounding $\gamma$ to lie below $\bar{\gamma}$. While they do not require the sequences $\left(\lambda_{t}\right)^{*}$ to be summable, they essentially assume that the sum grows lower than the discount factor $\beta$. Messner et al. (2011) take a different route. Instead of using the contraction mapping theorem they use monotone methods to prove existence of a Bellman operator and they do not need boundedness in their approach.

\subsection{Recursive multiplier formulation}

We now define a functional equation similar to the one in Marcet and Marimon (1994) and prove that the value function of the problem gives the value of the social planner's problem. The following functional equation defines the social planning problem in recursive multiplier form

$$
F(\gamma, x, s)=\sup _{\psi \in \Psi} \inf _{\lambda \geqslant 0} \sum_{a \in \operatorname{supp}(\psi)} \psi(a)\left[r(x, a, s)+\sum_{i=1}^{I}\left(\gamma^{i} g^{i}(x, a, s)+\lambda_{a}^{i}\left(g^{i}(x, a, s)-\bar{g}_{1}^{i}\right)\right)+\beta E_{s} F\left(\gamma+\lambda_{a}, x^{\prime}, s^{\prime}\right)\right]
$$

subject to

$$
x^{\prime}=\zeta(x, a, s)
$$




$$
\begin{aligned}
& p(x, a, s) \geqslant 0, \quad \forall a \in \operatorname{supp}(\psi), \\
& \gamma+\lambda_{a} \leqslant \bar{\gamma}, \quad \forall a \in \operatorname{supp}(\psi) .
\end{aligned}
$$

The following theorem guarantees the existence of a unique solution.

Theorem 1. Under Assumptions 1 and 2 the functional equation (10) has a unique solution in the space of bounded functions $F$ : $\bigotimes_{i=1}^{I}\left[0, \bar{\gamma}_{i}\right] \times \mathcal{X} \times \mathcal{S} \rightarrow \mathbb{R}, s \in \mathcal{S}$.

With our strong Assumption 2 we are able to give a simple proof to make our paper self-contained.

For $f: \bigotimes_{i=1}^{I}\left[0, \bar{\gamma}_{i}\right] \times \mathcal{X} \times \mathcal{S} \rightarrow \mathbb{R}$, consider the operator

$$
\begin{aligned}
& T f(\gamma, x, s)=\max _{\psi \in \Psi} \min _{\lambda \in[0, \bar{\lambda}]^{I}} \sum_{a \in \operatorname{supp}(\psi)} \psi(a)\left[r(x, a, s)+\sum_{i}\left(\gamma^{i} g^{i}(x, a, s)+\lambda_{a}^{i}\left(g^{i}(x, a, s)-\bar{g}_{1}^{i}\right)\right)+\beta E_{s} f\left(\gamma+\lambda_{a}, x^{\prime}, s^{\prime}\right)\right] \\
& \text { subject to } \\
& x^{\prime}=\zeta(x, a, s), \\
& \quad p(x, a, s) \geqslant 0, \quad \forall a \in \operatorname{supp}(\psi), \\
& \gamma+\lambda_{a} \leqslant \bar{\gamma}, \quad \forall a \in \operatorname{supp}(\psi) .
\end{aligned}
$$

We apply the contraction mapping theorem and verify Blackwell's sufficient conditions monotonicity (M) and discounting (D) (see e.g. Stokey and Lucas, 1989, Theorem 3.3).

(M) Given bounded $f^{1}$ and $f^{2}$ with $f^{1}(\gamma, x, s) \leqslant f^{2}(\gamma, x, s)$ for all $(\gamma, x, s)$, let $\left(\psi^{1}, \lambda^{1}\right)$ be in the arg-max of $T_{\bar{\lambda}} f^{1}(\gamma, x)$. Clearly,

$$
\begin{aligned}
T f^{1}(\gamma, x, s) \leqslant & \min _{\lambda: \gamma+\lambda \leqslant \bar{\gamma}} \sum_{a \in \operatorname{supp}\left(\psi^{1}\right)} \psi^{1}(a)\left[r(x, a, s)+\sum_{i}\left(\gamma^{i} g^{i}(x, a, s)+\lambda_{a}^{i}\left(g^{i}(x, a, s)-\bar{g}_{1}^{i}\right)\right)\right. \\
& \left.+\beta E_{s} f^{2}\left(\gamma+\lambda_{a}, x^{\prime}\right)\right] \\
\leqslant & T f^{2}(\gamma, x, s) .
\end{aligned}
$$

(D) Substituting yields $T_{\bar{\lambda}}(f+a)=T_{\bar{\lambda}}(f(\gamma, x)+a)=T_{\bar{\lambda}} f+\beta a$.

Having established the existence of a solution of the functional equation (10) raises the question how this solution relates to the solution of the original problem. This turns out to be somewhat intricate. Not because we bound $\gamma$ to lie below $\bar{\gamma}$ but for a different reason which we illustrate with a simple example.

\subsection{Policy- and value-functions}

Unfortunately, while the value function $F$ is well defined and continuous, the set of arguments that maximize $F$ might contain infinitely many solutions. While it is true that if a solution to original saddle point problem exists that this also solves (9), the converse is false, in the sense that there can be many solutions to (9) that do not solve the saddle point problem. This was first observed by Messner and Pavoni (2004). The problem has nothing to do with infinite horizon or uncertainty but is simply caused by the fact that one cannot recover policies from correspondences in a min-max problem. One can illustrate the problem with a trivial two-period example.

\subsubsection{Simple example}

Suppose we want to solve the following problem

$$
\begin{aligned}
& V=\max _{\left(a_{1}, a_{2}\right) \in[0,1]^{2}}-a_{1}-a_{2} \\
& \text { subject to } \quad a_{1}+a_{2} \geqslant 1 .
\end{aligned}
$$

The value of the corresponding Lagrangian can be obtained recursively (or by backward induction). We can write

$$
\begin{aligned}
& V_{2}(\lambda)=\max _{a_{2} \in[0,1]}\left(-a_{2}+\lambda a_{2}\right), \\
& V_{1}=\max _{a_{1} \in[0,1]} \min _{\lambda \geqslant 0}\left(-a_{1}+\lambda\left(a_{1}-1\right)+V_{2}(\lambda)\right)
\end{aligned}
$$


and get the correct value of the saddle point

$$
V_{1}=\max _{\left(a_{1}, a_{2}\right) \in[0,1]^{2}} \min _{\lambda \geqslant 0}\left(-a_{1}-a_{2}\right)+\lambda\left(a_{1}+a_{2}-1\right) .
$$

However, one cannot recover the correct policies from this if one takes the Lagrange multiplier as a state variable: The correspondence solving the problem in the second period is given by

$$
a_{2}(\lambda)=\arg \max _{a_{2} \in[0,1]}\left(-a_{2}+\lambda a_{2}\right)= \begin{cases}0, & \lambda<1, \\ {[0,1],} & \lambda=1, \\ 1, & \lambda>1 .\end{cases}
$$

So clearly, one element of the recursive problem's arg-max is $a_{1}=1 / 2, \lambda=1$ and $a_{2}=0$. But this is not a feasible point. Another element of the recursive problem's solution is $a_{1}=1 / 2, \lambda=1$ and $a_{2}=1$. This is a feasible point, but obviously suboptimal.

The recursive formulation has solutions which are infeasible and has solutions that are feasible but suboptimal. However note that in the above problem the solution to the optimization problem is in the correspondence of possible solutions to the recursive formulations.

A somewhat unrelated notational issue concerns how to properly define the arguments of a max-min problem. If we consider the original problem

$$
\max _{\left(a_{1}, a_{2}\right) \in[0,1]^{2}} \min _{\lambda \geqslant 0}\left(-a_{1}-a_{2}\right)+\lambda\left(a_{1}+a_{2}-1\right),
$$

then in the solution $\lambda$ is implicitly a function of the action $\left(a_{1}, a_{2}\right)$, where this function is

$$
\lambda(a)= \begin{cases}\infty & \text { if } a_{1}+a_{2}<1, \\ 1 & \text { if } a_{1}+a_{2}=1, \\ 0 & \text { if } a_{1}+a_{2}>1,\end{cases}
$$

and this function serves to enforce the constraint. In this case, $a_{1} \in[0,1]$ and $a_{2}=1-a_{1}$ is a solution to this problem and the value of the multiplier at the solution is of course $\lambda=1$. However, if we consider the min-max version of this problem,

$$
\min _{\lambda \geqslant 0} \max _{\left(a_{1}, a_{2}\right) \in[0,1]^{2}}\left(-a_{1}-a_{2}\right)+\lambda\left(a_{1}+a_{2}-1\right),
$$

then $\lambda=1, a_{1} \in[0,1]$ and $a_{2}=1-a_{1}$ is a solution to this problem but it is not the only one. In particular, with $\lambda=1$ any choices of $a_{1}$ and $a_{2}$ are solutions to the problem, including $a_{1}=a_{2}=1$ and $a_{1}=a_{2}=0$. Hence, one cannot enforce the constraint in a min-max version of the problem. With convexity, we can apply the max-min theorem and the value of the two problems is identical, however the solution to the first problem is the 'correct' solution. ${ }^{5}$ It will therefore be useful to make the following definition. We say that $(\bar{x}, \bar{y}) \in \arg \max _{x} \min _{y} f(x, y)$ if $\bar{x} \in \arg \max _{x}\left[\min _{y} f(x, y)\right]$, if $\bar{y} \in \arg \min y f(\bar{x}, y)$ and if $\bar{y} \in \arg \min _{y}\left[\max _{x} f(x, y)\right]$.

Both of these issues arise because of the lack of strict concavity of our problem. If the solution is unique, then neither is a problem.

\subsection{Principle of optimality}

We are given a solution $F^{*}$ to the functional equation (10). In order to construct a policy which solves the saddle point problem (and therefore the original maximization problem), using the above convention about the arg-max-min, we can define the correspondence

\footnotetext{
5 To understand the need for convexity of the constraint set in order to interchange the max and the min operators, consider the simple matching pennies game. In the deterministic version of this game $x$ and $y$ are elements of $[0,1]$ and the objective is $f(x, y)=-(x-y)^{2}$. In this case

$$
\max _{x} \min _{y} f(x, y)<\min _{y} \max _{x} f(x, y) .
$$

When we allow players to randomize, then the constraint set is convex. Let $x$ and $y$ denote the probabilities of playing 0 for each of the two players, $x$ and
} $y$ are elements of $[0,1]$, and the objective function becomes

$$
f(x, y)=-(1-x) y-x(1-y) .
$$

In this case

$$
\max _{x} \min _{y} f(x, y)=\min _{y} \max _{x} f(x, y)
$$




$$
\begin{aligned}
C(\gamma, x, s)= & \arg \max _{\psi} \min _{\lambda} \sum_{a \in \operatorname{supp}(\psi)} \psi(a) \\
& \times\left[r(x, a, s)+\sum_{i=1}^{I}\left(\gamma^{i} g^{i}(x, a, s)+\lambda_{a}\left(g^{i}(x, a, s)-\bar{g}_{1}^{i}\right)\right)+\beta E_{s} F^{*}\left(\gamma+\lambda_{a}, x^{\prime}, s^{\prime}\right)\right]
\end{aligned}
$$

subject to

$$
\begin{aligned}
& x^{\prime}=\zeta(x, a, s), \\
& p(x, a, s) \geqslant 0, \quad \forall a \in \operatorname{supp}(\psi), \\
& \gamma+\lambda_{a} \leqslant \bar{\gamma}, \quad \forall a \in \operatorname{supp}(\psi) .
\end{aligned}
$$

In this subsection, we want to prove the following theorem.

Theorem 2. Under Assumption 2, given solutions to the recursive problem, $\left(F^{*}, C\right)$, there exists a sequence $\left(\psi^{*}\left(h^{t}\right), \lambda^{*}\left(h^{t}\right)\right)$ with $\left(\psi^{*}\left(h^{t}\right), \lambda^{*}\left(h^{t}\right)\right) \in C\left(\gamma\left(\lambda_{0}, \ldots, \lambda_{t-1}\right), x\left(h^{t}\right), s_{t}\right)$ for all $h^{t}$ that solves the saddle point problem (9). Moreover, the value of the saddle point problem at $(\gamma, x, s)$ is given by $F^{*}(\gamma, x, s)$.

The proof is quite similar to Bellman's principle of optimality (see e.g. Stokey and Lucas, 1989, Section 4.1).

Proof. We first prove that if there exists a solution to the saddle point problem, the value must coincide with $F^{*}$. For this define

$$
\begin{aligned}
F_{\tau}\left(\gamma_{0}, x_{0}, s_{0}\right)= & \sup _{\psi_{0}, \ldots, \psi_{\tau}\left(\lambda_{0}, \ldots, \lambda_{\tau}\right) \geqslant 0} \inf _{0}^{\psi_{0}, \ldots, \psi_{\tau}} \sum_{t=0}^{\tau} \beta^{t} \\
& \times\left(r\left(x_{t}, a_{t}, s_{t}\right)+\sum_{i}\left(\lambda_{t, a_{t}}^{i}\left(g^{i}\left(x_{t}, a_{t}, s_{t}\right)-\bar{g}_{1}^{i}\right)+g^{i}\left(x_{t}, a_{t}, s_{t}\right)\left(\gamma_{0}^{i}+\sum_{n=0}^{t-1} \lambda_{n}^{i}\right)\right)\right) \\
& +E_{0}^{\psi_{0}, \ldots, \psi_{\tau}} \beta^{\tau+1} F^{*}\left(\left(\gamma_{0}+\sum_{n=0}^{\tau} \lambda_{n}\right), x_{\tau+1}, s_{\tau+1}\right)
\end{aligned}
$$

subject to $\operatorname{supp}\left(\psi_{t}\right) \subset \mathcal{A}\left(h^{t}\right) \quad$ for all $h^{t}, \quad x_{0}, s_{0}$ given,

$$
\gamma_{0}+\sum_{n=0}^{\tau} \lambda_{n} \leqslant \bar{\gamma} .
$$

Note that for $\tau=1$ we obtain

$$
\begin{aligned}
& \begin{aligned}
F_{1}\left(\gamma_{0}, x_{0}, s_{0}\right)= & \sup _{\psi_{0}, \psi_{1}} \inf _{\lambda_{0}, \lambda_{1}} E_{0} \sum_{t=0}^{1} \beta^{t} \sum_{a \in \operatorname{supp}\left(\psi_{t}\right)} \psi_{t}(a) \\
& \times\left(r\left(x_{t}, a, s_{t}\right)+\sum_{i}\left(\lambda_{a, t}^{i}\left(g^{i}\left(x_{t}, a, s_{t}\right)-\bar{g}^{i}\right)+g^{i}\left(x_{t}, a, s_{t}\right)\left(\gamma_{0}^{i}+\sum_{n=0}^{t-1} \lambda_{n}^{i}\right)\right)\right) \\
& +E_{0} \beta^{2} F^{*}\left(\left(\gamma_{0}+\sum_{n=0}^{1} \lambda_{n}\right), x\left(h^{3}\right), s_{3}\right)
\end{aligned} \\
& \text { subject to } \operatorname{supp}\left(\psi_{t}\right) \subset \mathcal{A}\left(h^{t}\right) \quad \text { for all } h^{t}, \quad x_{0}, s_{0} \text { given, } \\
& \gamma_{0}+\sum_{n=0}^{1} \lambda_{n} \leqslant \bar{\gamma} .
\end{aligned}
$$

By Fan's min-max theorem and since the problem is additively separable this can be rewritten as

$$
\begin{aligned}
F_{1}\left(\gamma_{0}, x_{0}, s_{0}\right)= & \operatorname{supinf}_{\psi_{0}} E_{\lambda_{0}} \sum_{a \in \operatorname{supp}\left(\psi_{0}\right)} \psi_{0}(a) \\
& \times\left(r\left(x_{0}, a, s_{0}\right)+\sum_{i}\left(\lambda_{0 a}^{i}\left(g^{i}\left(x_{0}, a, s_{0}\right)-\bar{g}_{1}^{i}\right)+g^{i}\left(x_{0}, a, s_{t}\right) \gamma_{0}^{i}\right)\right)
\end{aligned}
$$




$$
\begin{aligned}
& +\beta E_{0} \operatorname{supinf}_{\psi_{1}} \sum_{\lambda_{1}} \psi_{a \in \operatorname{supp}\left(\psi_{1}\right)} \psi_{1}(a) \\
& \times\left(r\left(x_{1}, a, s_{1}\right)+\sum_{i}\left(\lambda_{1 a}^{i}\left(g^{i}\left(x_{1}, a, s_{1}\right)-\bar{g}_{1}^{i}\right)+g^{i}\left(x_{1}, a, s_{1}\right)\left(\gamma_{0}^{i}+\lambda_{0}^{i}\right)\right)\right) \\
& +E_{0} \beta^{2} F^{*}\left(\left(\gamma_{0}+\sum_{n=0}^{1} \lambda_{n}\right), x\left(h^{2}\right), s_{3}\right)
\end{aligned}
$$

subject to $\operatorname{supp}\left(\psi_{t}\right) \subset \mathcal{A}\left(h^{t}\right) \quad$ for all $h^{t}, \quad x_{0}, s_{0}$ given,

$$
\gamma_{0}+\sum_{n=0}^{1} \lambda_{n} \leqslant \bar{\gamma} .
$$

Since this amounts to applying our recursive operator twice, clearly, $F_{1}=F^{*}$ and in fact by induction, $F_{\tau}\left(\gamma_{0}, x_{0}, s_{0}\right)=$ $F^{*}\left(\gamma_{0}, x_{0}, s_{0}\right)$ for each $\tau=1,2, \ldots$.

On the other hand, we can write

$$
\begin{aligned}
F_{\tau}\left(\gamma_{0}, x_{0}, s_{0}\right)= & \sup _{\psi_{0}, \ldots, \psi_{\tau} \lambda_{0}, \ldots, \lambda_{\tau}} \inf _{0}^{\psi_{0}, \ldots, \psi_{\tau}} \sum_{t=0}^{\tau} \beta^{t} \\
& \times\left(r\left(x_{t}, a_{t}, s_{t}\right)+\sum_{i=1}^{I}\left(\gamma_{0}^{i} g^{i}\left(x_{t}, a_{t}, s_{t}\right)+\lambda_{t, a_{t}}^{i}\left(\sum_{n=0}^{\tau-t} \beta^{n} g^{i}\left(x_{t+n}, a_{t+n}, s_{t+n}\right)-\bar{g}_{1}^{i}\right)\right)\right) \\
& +E_{0}^{\psi_{0}, \ldots, \psi_{\tau}} \beta^{\tau+1} F^{*}\left(\left(\gamma_{0}+\sum_{n=0}^{\tau} \lambda_{n}\right), x_{\tau+1}, s_{\tau+1}\right)
\end{aligned}
$$

subject to $\operatorname{supp}\left(\psi_{t}\right) \subset \mathcal{A}\left(h^{t}\right) \quad$ for all $h^{t}, \quad x_{0}, s_{0}$ given,

$$
\gamma_{0}+\sum_{n=0}^{\tau} \lambda_{n} \leqslant \bar{\gamma} .
$$

Recall that the value of the saddle point is

$$
\begin{aligned}
L^{*}\left(\gamma_{0}, x_{0}, s_{0}\right)= & \sup _{\left(\psi_{t}\right)} \inf _{\left(\lambda_{t}\right)} E_{0}^{\psi} \sum_{t=0}^{\infty} \beta^{t} \\
& \times\left(r\left(x_{t}, a_{t}, s_{t}\right)+\sum_{i=1}^{I}\left(\gamma_{0}^{i} g^{i}\left(x_{t}, a_{t}, s_{t}\right)+\lambda_{t, a_{t}}^{i}\left(\sum_{n=0}^{\infty} \beta^{n} g\left(x_{t+n}, a_{t+n}, s_{t+n}\right)-\bar{g}_{1}^{i}\right)\right)\right) .
\end{aligned}
$$

By the same argument as above, for any $\tau>0$, this can be written as

$$
\begin{aligned}
L^{*}\left(\gamma_{0}, x_{0}, s_{0}\right)= & \sup _{\psi_{0}, \ldots, \psi_{\tau}} \inf _{\lambda_{0}, \ldots, \lambda_{\tau}} E_{0}^{\psi} \sum_{t=0}^{\tau} \beta^{t} \\
& \times\left(r\left(x_{t}, a_{t}, s_{t}\right)+\sum_{i=1}^{I}\left(\gamma_{0}^{i} g^{i}\left(x_{t}, a_{t}, s_{t}\right)+\lambda_{t, a_{t}}^{i}\left(\sum_{n=0}^{\tau-t} \beta^{n} g^{i}\left(x_{t+n}, a_{t+n}, s_{t+n}\right)-\bar{g}_{1}^{i}\right)\right)\right) \\
& +\sup _{\psi_{\tau+1}, \ldots} \inf _{\tau+1} E_{0}^{\psi_{0}, \ldots, \psi_{\tau}} \sum_{t=\tau+1}^{\infty} \beta^{t} \\
& \times\left(r\left(x_{t}, a_{t}, s_{t}\right)+\sum_{i=1}^{I}\left(\left(\gamma_{0}^{i}+\sum_{j=0}^{\tau} \lambda_{j}^{i}\right) g^{i}\left(x_{t}, a_{t}, s_{t}\right)+\lambda_{t, a_{t}}^{i}\left(\sum_{n=0}^{\infty} \beta^{n} g^{i}\left(x_{t+n}, a_{t+n}, s_{t+n}\right)-\bar{g}_{1}^{i}\right)\right)\right) .
\end{aligned}
$$

If for the optimal $\lambda^{*}$ there exist a $\tau$ and an $i$ such that $\gamma_{0}^{i}+\sum_{t=0}^{\tau} \lambda_{t}^{i *}>\bar{\gamma}_{i}, \gamma_{0}^{i}+\sum_{t=0}^{\tau-1} \lambda_{t}^{i *} \leqslant \bar{\gamma}_{i}$, by Assumption 2, we obtain the same value of the saddle point by replacing $\lambda^{*}$ by $\tilde{\lambda}$ where $\tilde{\lambda}_{t}=\lambda_{t}^{*}$ for $t<\tau, \tilde{\lambda}_{\tau}^{i}=\bar{\gamma}_{i}-\sum_{t=0}^{\tau-1} \tilde{\lambda}^{i}$ and $\tilde{\lambda}_{t}^{i}=0$ for $t>\tau$. Therefore we can assume that the solution to this saddle point problem satisfies $\sum_{t=0}^{\infty} \lambda^{*} \leqslant \gamma$.

Since $F^{*}$ is bounded, the reward functions are bounded and since the optimal multipliers to the saddle point problem are bounded (see Rustichini, 1998) it follows that 


$$
\lim _{\tau \rightarrow \infty} L^{*}\left(\gamma_{0}, x_{0}, s_{0}\right)-F_{\tau}\left(\gamma_{0}, x_{0}, s_{0}\right)=0 .
$$

Therefore the value of the saddle point must be equal to $F^{*}$.

Furthermore the solution to the saddle point equation is certainly feasible for the recursive social planner's problem. Since it gives the optimal value, $F^{*}$, it must satisfy $\left(\psi_{t}^{*}, \lambda_{t}^{*}\right) \in C\left(\gamma\left(\lambda_{0}^{*}, \ldots, \lambda_{t-1}^{*}\right), x\left(h^{t}\right), s_{t}\right)$ for all $t$.

\section{Recovering policy functions}

The previous theorem shows that among the (possibly many) solutions to the functional equation (10) there is one which also solves our original problem. The question still remains how to find it. In this section, we explicitly construct a solution to the maximization problem, using the solution to the functional equation (10).

We proceed in two steps. First we consider the general problem, here it will become clear that we are mixing the promised utility approach with the recursive multiplier approach of Marcet and Marimon (1994) to obtain well-defined policy functions. ${ }^{6}$ We then make an assumption on the solution set of the problem which simplifies the analysis considerably and allows us to derive a simple characterization of the solution.

\subsection{The general approach}

By the maximum principle it is easy to see that the correspondence $C($.$) , as defined in the previous section, is upper$ hemi-continuous. We can therefore define

$$
A(\gamma, x, s)=\{(a, \lambda): \exists(\psi, \lambda) \in C(\gamma, x, s): a \in \operatorname{supp}(\psi)\} .
$$

Note that all $(a, \lambda) \in A(\gamma, x, s)$ yield the same value of the objective function (of the social planner's problem, given $\gamma$ ). Note also that if $\left(\psi_{t}, \lambda_{t}\right)_{t=0}^{\infty}$ solves the saddle point problem, then in fact each $a_{0} \in \operatorname{supp}\left(\psi_{0}\right)$ is feasible and optimal. As the example in Section 3.3.1 shows, the problem comes about because one needs to establish a link between periods. It is well known that one way to do this is to carry agents' promised utilities.

For a given 'state', $(\gamma, x, s)$, there might be several possible utilities that can be generated by the several possible actions $(a, \lambda) \in A(\gamma, x, s)$ and by the randomizations over these actions. In a slight abuse of notation, we use $G$ both for the function and the correspondence of possible utility levels and collect these in a set $G(\gamma, x, s)$. The correspondence, $G$, from the state to the set of possible (i.e. feasible) utilities, is defined by the following system:

$$
\begin{aligned}
G(\gamma, x, s)= & \operatorname{conv}\left\{\left(G^{0}, G^{1}, \ldots, G^{I}\right) \in \mathbb{R}^{I+1}: \exists(a, \lambda) \in A(\gamma, x, s)\right. \\
& \exists\left(G_{s^{\prime}}^{0 \prime}, \ldots, G_{s^{\prime}}^{I \prime}\right) \in G\left(\gamma+\lambda, \zeta(x, a, s), s^{\prime}\right) \text { for all } s^{\prime}, \\
& G^{0}=r(x, a, s)+\beta E_{s} G^{0 \prime}, \\
& G^{1}=g^{1}(x, a, s)+\beta E_{s} G^{1 \prime}, G^{1} \geqslant \bar{g}_{1}^{1} \\
& \vdots \\
& \left.G^{I}=g^{I}(x, a, s)+\beta E_{s} G^{I \prime}, G^{I} \geqslant \bar{g}_{1}^{I}\right\},
\end{aligned}
$$

where $\operatorname{conv}(A)$ denotes the convex hull of a set $A$.

For each admissible state, $\gamma, x, s$, the set $G(\gamma, x, s)$ is a non-empty and convex subset of the $I$-dimensional hyperplane with normal-vector $\left(1, \gamma_{1}, \ldots, \gamma_{I}\right)$. That is $G^{0}+\sum_{i=1}^{I} \gamma_{i} G^{I}=$ const for all $\left(G^{0}, \ldots, G^{I}\right) \in G(\gamma, x, s)$. If the problem is strictly concave, this set will contain only one point - in general, however, it has 'full' dimension $I$.

Obviously there must be a (possible set-valued map) from $(\gamma, x, s)$ and a given $G \in G(\gamma, x, s)$ to randomized actions, $\psi$,

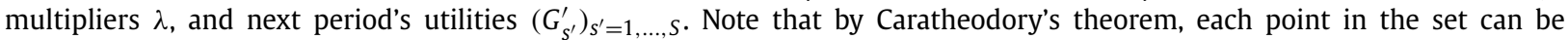
obtained by randomizing over at most $I+1$ actions $a$. If we define the state space to contain utilities promised in the last period as well as $\gamma, x$ and $s$, we can easily construct policies that are optimal and feasible. Given an enlarged state $(\gamma, x, s)$ and $G \in G(\gamma, x, s)$ we now say that a policy consists of $\psi, \lambda$ as well as $\left(G_{s^{\prime}}^{\prime}\right)_{s^{\prime}=1, \ldots, S}$ that is consistent with the requirements above, i.e.

$$
G^{0}=r(x, a, s)+\beta E_{s} G^{0 \prime}, \quad \ldots, \quad G^{I}=g^{I}(x, a, s)+\beta E_{s} G^{I \prime} \geqslant \bar{g}_{1}^{I} .
$$

In order to tie this in with our results in the previous sections, it is useful, however, to choose a slightly different approach. This appears more complicated at first, but will simplify things greatly once we make additional assumptions. We denote by $\widetilde{G}(\gamma, x, s)$ all utilities that can be obtained at a state $(\gamma, x, s)$ without using randomization in the current period,

\footnotetext{
6 In Section 4 below we make explicit how our approach compares to the promised utility approach.

7 Formally we now enlarge the state space to contain agents' utilities, however, when we refer to the state, we mean the current multiplier, current physical state and current shock.
} 
i.e. $G(\gamma, x, s)=\operatorname{conv}(\widetilde{G}(\gamma, x, s))$ and each $G \in \widetilde{G}(\gamma, x, s)$ is associated with a degenerate probability distribution over current actions. A non-standard way of deriving policies now is that a policy specifies $(a, \lambda) \in A(\gamma, x, s)$ as well as $G_{s^{\prime} k}^{\prime}$ and $\pi_{s^{\prime}, k}$, $s^{\prime}=1, \ldots, S$ and $k=1, \ldots, I$ with the property that each $G_{s^{\prime} k}^{\prime} \in \widetilde{G}\left(\gamma+\lambda, x^{\prime}, s^{\prime}\right)$ and that

$$
\begin{aligned}
& G^{0}=r(x, a, s)+\beta E_{s} \sum_{k} \pi_{s^{\prime} k} G_{s^{\prime} k}^{\prime 0}, \\
& G^{1}=g^{1}(x, a, s)+\beta E_{s} \sum_{k} \pi_{s^{\prime} k} G_{s^{\prime} k}^{1 \prime} \geqslant \bar{g}_{1}^{1}, \\
& \vdots \\
& G^{I}=g^{I}(x, a, s)+\beta E_{s} \sum_{k} \pi_{s^{\prime} k} G_{s^{\prime} k}^{I \prime} \geqslant \bar{g}_{1}^{I} .
\end{aligned}
$$

In other words, the policy today is given by a non-randomized action today and a randomization over all actions tomorrow, shock by shock. By Caratheodory's theorem one only has to randomize over at most $I$ actions in each state tomorrow. In order to make clear that we randomize over a fixed set of continuation utilities, we now use $\pi$ to denote the probabilities of these randomization, instead of $\psi$, the randomization over actions which are obviously implied by this.

\subsection{A special case}

A special case obtains when the sets $G(\gamma, x, s)$ are simple in the sense that they can be written as the convex hull of a finite number of points. In this case, we do not have to introduce an additional continuous state variable.

To illustrate this, we now make the following strong assumptions on the solution set of the problem.

Assumption 3. For each admissible $\gamma, x \in \mathcal{X}, x \in \mathcal{S}$, the set $G(\gamma, x, s)$ is a convex polytope, i.e. the convex hull of a finite set of points.

Note that for the case $I=1$, i.e. the case of one agent and one principal, the assumptions always hold since $G(\gamma, x, s)$ is simply a closed interval. We consider this case in some detail in the next subsection. For more than one agent, this obviously depends on the formulation of the problem. Even if the set of actions $\mathcal{A}$ is finite, it is not guaranteed that $G(\gamma, x, s)$ will be a polytope - it is quite likely though. However, if the true solution set is not a polytope, the most obvious approach to approximate it numerically is to use a polytope. In this case the number of points just needs to be sufficiently large in order to obtain a good approximation.

It is clear that with this assumption, the state does not have to be enlarged by an infinite set but simply by a finite set of discrete states. For a given set of points, $P$, whose convex hull forms a polytope, let $\mathcal{V}(P)$ denote the (finite set) of extreme points (i.e. vertices) of this polytope, i.e. the smallest set of points for which $\operatorname{conv}(P)=\operatorname{conv}(\mathcal{V}(P))$. Furthermore, let $g(x, a, s)=\left(g^{0}(x, a, s), \ldots, g^{I}(x, a, s)\right)$ and let $\mathcal{F}=\left\{\left(G_{0}, \ldots, G_{I}\right): G_{1} \geqslant \bar{g}_{1}^{1}, \ldots, G_{I} \geqslant \bar{g}_{1}^{I}\right\}$ be the set of utilities satisfying the incentive constraint (i.e. feasible utilities). We define a correspondence $\mathcal{G}$, mapping the current state to a finite set of points, $\mathcal{G}:[0, \infty)^{I} \times \mathcal{X} \times \mathcal{S} \Rightarrow \mathbb{R}^{I+1}$, by requiring that it satisfies the following functional equation

$$
\begin{aligned}
& \mathcal{G}(\gamma, x, s)=\mathcal{V}\left[\bigcup_{(a, \lambda) \in A(\gamma, x, s)}\left(\mathcal{F} \cap \operatorname{conv}\left(\{g(x, a, s)\}+\beta \sum_{s^{\prime}} \Pi\left(s, s^{\prime}\right) \mathcal{G}\left(\gamma+\lambda, x^{\prime}, s^{\prime}\right)\right)\right)\right], \\
& x^{\prime}=\zeta(x, a, s) .
\end{aligned}
$$

In this equation the sum of sets is the Minkowski sum with $\alpha A+\beta B=\{\alpha a+\beta b: a \in A, b \in B\}$.

A solution exists precisely when $G(\gamma, x, s)$ is a polytope - in this case $\mathcal{G}(\gamma, x, s)$ is simply the set of its extreme points. The advantage of writing it like this (as opposed to the system that defined $G(\gamma, x, s)$ in the previous subsection) is that it becomes evident, that these sets can be constructed relatively easily from the knowledge of the correspondence $A($.$) .$

When a solution to (12) exists an optimal policy can be constructed as follows. For each $v \in \mathcal{G}(\gamma, x, s)$, define

$$
\bar{A}(\gamma, x, s, v)=\left\{(a, \lambda) \in A(\gamma, x, s),\left(\pi_{1}, \ldots, \pi_{s}\right): v=g(x, a, s)+\beta \sum_{s^{\prime}} \Pi\left(s, s^{\prime}\right) \sum_{v^{\prime} \in \mathcal{G}\left(\gamma+\lambda, x^{\prime}, s^{\prime}\right)} \psi_{s^{\prime}}\left(v^{\prime}\right) v^{\prime}\right\} .
$$

This gives a quasi-policy function in the following sense. The current state now consists of $\gamma, x, s$ as well as a $v \in \mathcal{G}(\gamma, x, s)$. The policy prescribes the current non-randomized action, the current multiplier, $\lambda$ as well as (a possibly degenerate) randomization over next periods states (which in effect means a randomization over actions in the next period). There is in general no guarantee that $\bar{A}$ is single-valued, but each selection will produce an optimal policy.

Note that in general one needs to choose a randomization for each shock $s^{\prime}$ in the next period, i.e. $\pi$ will differ across shocks next period. We will show below that for the special case of one agent and one principal this is not the case there the same randomization can be chosen for each shock. It is clear why in this general framework this cannot be 
expected - there is no guarantee that the number of vertices is the same for each shock next periods, therefore it is obviously impossible to find a randomization that does not depend on the shock.

Note that there is only randomization if today at least one incentive constraint is binding.

Theorem 3. There is an optimal solution $(a, \lambda, \pi)$ with each $\pi_{s}$ being a degenerate probability distribution if $\left(v_{1}, \ldots, v_{I}\right) \gg$ $\left(\bar{g}_{1}^{1}, \ldots, \bar{g}_{1}^{I}\right)$.

To prove the result, observe that in order for $v$ to be an element of

$$
\mathcal{V}\left[\bigcup_{(a, \lambda) \in A(\gamma, x, s)}\left(\{g(x, s, a)\}+\beta \sum_{s^{\prime}} \Pi\left(s, s^{\prime}\right) \mathcal{G}\left(\gamma+\lambda, x^{\prime}, s^{\prime}\right)\right)\right]
$$

it has to be generated by an action next period that is not randomized - each vertex of the sum of polytopes must be the sum of vertices (see e.g. Fukuda, 2004). The result does not seem completely obvious since one might have thought that it could be optimal to randomize in two periods if an IC constraint is only binding this period.

From a computational aspect, polytopes are easy to deal with. There is a sizable literature on polyhedral computation. For example, Fukuda (2004) discusses an algorithm for the Minkowski addition of polytopes that can be applied to very large scale problems. For solving problems with large $I$, the key problem lies in solving for the correspondence $A$, if the true solution set of utilities is a polytope, the computation of $\mathcal{G}($.) and $\bar{A}$ is quite straightforward. If one is to solve the functional equation (12) iteratively, one can easily see if the number of extreme points grows with each iteration or if eventually this stabilizes.

\subsection{A principal and one agent}

If there is only one agent (in addition to the principal), Assumption 2 is trivially satisfied and we can set up the problem somewhat more intuitively. Since $G(\gamma, x, s)$ is at most an interval the sets $\mathcal{G}(\gamma, x, s)$ contain at most two points. We can characterize these two points as the one that maximizes the agent's utility and then one that minimizes the agents utility and denote them by $G^{H}(\gamma, x, s)$ and $G^{L}(\gamma, x, s)$. Furthermore the probabilities over next periods' continuation payoffs (and hence next periods actions) can now be chosen independently of the shock.

We can consider the following functional equation:

$$
\begin{aligned}
& G^{L}(\gamma, x, s)=\min _{(\underline{a}, \underline{\lambda}) \in A(\gamma, x, s), \pi \in[0,1]} g(x, \underline{a}, s)+\beta E_{s}\left(\pi G^{L}\left(\gamma+\underline{\lambda}, \underline{x}^{\prime}, s^{\prime}\right)+(1-\pi) G^{H}\left(\gamma+\underline{\lambda}, \underline{x}^{\prime}, s^{\prime}\right)\right), \\
& G^{H}(\gamma, x, s)=\max _{(\bar{a}, \bar{\lambda}) \in A(\gamma, x, s), \pi \in[0,1]} g(x, \bar{a}, s)+\beta E\left(\pi G^{L}\left(\gamma+\bar{\lambda}, \bar{x}^{\prime}, s^{\prime}\right)+(1-\pi) G^{H}\left(\gamma+\bar{\lambda}, \bar{x}^{\prime}, s^{\prime}\right)\right)
\end{aligned}
$$

subject to

$$
\underline{x}^{\prime}=\zeta\left(x, \underline{a}, s^{\prime}\right), \quad \bar{x}^{\prime}=\zeta\left(x, \bar{a}, s^{\prime}\right), \quad G^{H}(\gamma, x, s) \geqslant \bar{g}_{1}, \quad G^{L}(\gamma, x, s) \geqslant \bar{g}_{1} .
$$

Given a solution $G^{H *}, G^{L *}$ to this functional equation we define

$$
\begin{aligned}
& \omega_{L}^{*}(\gamma, x, s)=\arg \min _{(a, \lambda) \in A(\gamma, x, s), \pi \in[0,1]} g(x, a, s)+\beta E\left(\pi G^{L *}\left(\gamma+\lambda, x^{\prime}, s^{\prime}\right)+(1-\pi) G^{H *}\left(\gamma+\lambda, x^{\prime}, s^{\prime}\right)\right) \\
& \text { subject to } x^{\prime}=\zeta(x, a, s)
\end{aligned}
$$

and

$$
\begin{aligned}
& \omega_{H}^{*}(\gamma, x, s)=\arg \max _{(a, \lambda) \in A(\gamma, x, s), \pi \in[0,1]} g(x, a, s)+\beta E\left(\pi G^{L *}\left(\gamma+\lambda, x^{\prime}, s^{\prime}\right)+(1-\pi) G^{H *}\left(\gamma+\lambda, x^{\prime}, s^{\prime}\right)\right) \\
& \text { subject to } x^{\prime}=\zeta(x, a, s) .
\end{aligned}
$$

Note that $\omega_{L}^{*}$ and $\omega_{H}^{*}$ are not policy functions in the classical sense. The policy should specify a randomization $\psi$ and a transition $\lambda$ as a function of the current state. But we have seen that this is not possible. Instead we change the timing and the state. The state now consists of $(\gamma, x, s)$ as well as an element of $\{H, L\}$. The timing now says that given the state, we choose a deterministic action, but also the randomization across actions next period. Note that this randomization will not be conditional on the realized shock next period - but this does not matter since the (IC) holds today.

By construction, these policies are feasible. The mapping could still be set-valued, but then we can simply pick any point in that set. Since the solution is feasible, the argument from Theorem 3 can be applied to show that it must also be optimal, i.e. an optimal solution to the original problem can be generated from $\omega$, given an initial $\gamma_{0}$ that ensures that the (PC) holds.

Finally, note that in the solution to these functional equations $\omega_{H}^{*}(\gamma, x, s)$ will trivially set $\pi=0$ so as to put the maximum probability onto the high continuation payoff for the agent. In the solution to $\omega_{L}^{*}(\gamma, x, s)$, it will be optimal to set $\pi=1$ so long as this does not lead to a violation of the incentive constraint that $G^{L}(\gamma, x, s) \geqslant \bar{g}_{1}$. If this constraint is violated at $\pi=1$, then it may be optimal to lower $\pi$ since this allows the solution to satisfy the incentive constraint at no additional cost to the overall value of the Lagrangian. 


\subsubsection{The optimal contract}

Given these constructions, we can now characterize the dynamics of the optimal contract in terms of $(\gamma, x, s)$ and an element of $\{H, L\}$.

In the initial period the initial physical state is $x_{0}$, and the promised utility $\bar{g}_{2}$ associated with the participation constraint must be satisfied. To do so, we select $\gamma_{0}$ by the requirement that

$$
E_{s} G^{H}\left(\gamma_{0}, x, s\right) \geqslant \bar{g}_{2} \geqslant E_{s} G^{L}\left(\gamma_{0}, x, s\right),
$$

and we select the initial lottery probabilities over $\{H, L\}, \zeta$ and $1-\zeta$, by the requirement that

$$
\bar{g}_{2}=\zeta E_{s} G^{H}\left(\gamma_{0}, x, s\right)+(1-\zeta) E_{s} G^{L}\left(\gamma_{0}, x, s\right) .
$$

This then determines the full initial state $(\gamma, x, s)$ and an element of $\{H, L\}$. Each period thereafter begins with this initial state.

Within each period thereafter the contract evolves as follows:

1. The element of $\{H, L\}$ determines which policy function $\omega_{H}^{*}$ and $\omega_{L}^{*}$ is used within the period.

2. The policy function $\omega_{i}$ determines a continuation triplet $\left(\gamma+\lambda, x^{\prime}, s^{\prime}\right)$ for next period and a probability $\pi$ for the lottery over elements of $\{H, L\}$. If the neither the participation or incentive constraint binds, then $\pi$ is simply 0 if the element is $H$ and 1 if the element is $L$. The participation constraint cannot bind if the element is $H$ and if it binds when the element is $L$ then $\pi$ will be less than one. If the incentive constraint binds, then $\pi$ is chosen so that

$$
g\left(x, a_{i}, s\right)+\beta E_{s}\left(\pi G^{L}\left(\gamma+\underline{\lambda}, \underline{x}^{\prime}, s^{\prime}\right)+(1-\pi) G^{H}\left(\gamma+\underline{\lambda}, \underline{x}^{\prime}, s^{\prime}\right)\right)=\bar{g}_{1},
$$

where $a_{i}$ is the minimizing element of the solution set for actions if $i=L$ and maximizing if $i=H$.

3. This lottery is undertaken at the end of the period and an element is selected. This determines the state tomorrow as $\left(\gamma+\lambda, x^{\prime}, s^{\prime}\right)$ and the element $a \in\{H, L\}$.

The key thing to note here is that the determination of the lottery probability $\pi$ is very mechanical and does not require solving an ex ante simultaneous equation problem such as would be the case under the utility approach, which we discuss next.

\subsection{Relation to 'promised utility' approach}

It is useful to connect the multiplier approach to the standard 'promised utility method', which was originally developed by Spear and Srivastava (1987), and where the state consists of the physical states as well as of an additional endogenous state variable, promised utility, $G$. This section is somewhat less formal. We focus on the case $I=1$ and we assume that the promised utility approach has a solution and that the resulting value function is differentiable. With this, we can demonstrate that the value of the Pareto problem must be identical to the value of the recursive problem of the previous section.

It is useful to change the timing and write value of the Pareto problem as a function of $(G, x)$ before the current shock $s$ is realized. In a Markov setup, we then need to carry around $s_{-}$as a state variable and we can write our Pareto problem recursively using the promised utility approach as

$$
\begin{aligned}
V\left(G, x, s_{-}\right)= & \max _{\left(\psi_{s}\right) \in \Psi^{S},\left(G_{s, a}^{\prime}\right)} \min _{\gamma \geqslant 0} \sum_{s \in \mathcal{S}} \Pi\left(s_{-}, s\right) \sum_{a \in \operatorname{supp}\left(\psi_{s}\right)} \psi_{s}(a) \\
& \times\left(r(x, a, s)+\beta V\left(G_{s, a}^{\prime}, x_{s}^{\prime}, s\right)+\gamma\left(g(x, a, s)+\beta G_{s, a}^{\prime}-G\right)\right)
\end{aligned}
$$

subject to

$$
\begin{aligned}
& x_{s}^{\prime}=\zeta\left(x, a_{s}, s\right), \\
& p(x, a, s) \geqslant 0, \quad \forall a \in \operatorname{supp}\left(\psi_{s}\right), \\
& g(x, a, s)+\beta G_{s, a}^{\prime} \geqslant \bar{g}_{1}, \quad \forall a \in \operatorname{supp}\left(\psi_{s}\right) .
\end{aligned}
$$

Note that we have included the promised utility of the agent $G$ through a Lagrangian multiplier formulation where $\gamma$ is the multiplier on this constraint. Obviously, at the optimal solution it must hold that $\gamma\left(g(x, a, s)+\beta G_{s, a}^{\prime}-G\right)=0$.

Assuming differentiability, each $G$ gives us a unique $\gamma(G)$, since by the envelope theorem $V_{G}\left(G, x, s_{-}\right)=-\gamma$. Unfortunately that the map is not invertible. For each $\gamma \in \Gamma \subset[0, \infty)$, we can define (assuming differentiability)

$$
\begin{aligned}
& V^{L}(\gamma, x, s)=\min \left\{V(G, x, s): V_{G}(G, x, s)=-\gamma\right\}, \\
& V^{H}(\gamma, x, s)=\max \left\{V(G, x, s): V_{G}(G, x, s)=-\gamma\right\} .
\end{aligned}
$$

We can also define the associated payoffs to the agent as 


$$
\begin{aligned}
& G^{L}(\gamma, x, s)=\arg \min \left\{V(G, x, s): V_{G}(G, x, s)=-\gamma\right\}, \\
& G^{H}(\gamma, x, s)=\arg \max \left\{V(G, x, s): V_{G}(G, x, s)=-\gamma\right\} .
\end{aligned}
$$

Note that we must have that for all $\gamma, x, s$,

$$
V^{L}(\gamma, x, s)+\gamma G^{L}(\gamma, x, s)=V^{H}(\gamma, x, s)+\gamma G^{H}(\gamma, x, s) .
$$

Given these functions, we can rewrite our recursive Pareto problem in terms of these functions, using the insight from before that the derivative of $V$ in the next period must be equal to $\gamma$ plus the value of the Lagrange parameter attached to this period's (IC). Writing $\gamma$ to denote the optimal $\gamma$ associated with $G$ we obtain

$$
\begin{aligned}
V\left(G, x, s_{-}\right)= & \max _{\left(a_{s}\right),\left(\pi_{s}\right)} \min _{\left(\lambda_{s}\right)} \sum_{s} \Pi\left(s_{-}, s\right) r\left(x_{s}, a_{s}, s\right) \\
& +\beta\left(\pi_{s} V^{L}\left(\gamma+\lambda_{s}, x_{s}^{\prime}\right)+\left(1-\pi_{s}\right) V^{H}\left(\gamma+\lambda_{s}, x_{s}^{\prime}\right)\right) \\
& +\gamma\left(g\left(x_{s}, a_{s}, s\right)+\beta\left(\pi_{s} G^{L}\left(\gamma+\lambda_{s}, x_{s}^{\prime}\right)+\left(1-\pi_{s}\right) G^{H}\left(\gamma+\lambda_{s}, x_{s}^{\prime}\right)\right)-G\right) \\
& +\lambda_{s}\left(g\left(x_{s}, a_{s}, s\right)+\beta\left(\pi_{s} G^{L}\left(\gamma+\lambda_{s}, x_{s}^{\prime}\right)+\left(1-\pi_{s}\right) G^{H}\left(\gamma+\lambda_{s}, x_{s}^{\prime}\right)\right)-\bar{g}_{1}\right) \\
\text { subject to } \quad x_{s}^{\prime} & =\zeta\left(x_{s}, a_{s}, s\right), \quad p\left(x_{s}, a_{s}, s\right) \geqslant 0, \quad \forall s .
\end{aligned}
$$

Note that the value $V(G, x, s)$ is linear in $G$ for all $\widetilde{G}$ that give the same value of $\gamma-$ i.e. we have that

$$
V\left(\widetilde{G}, x, s_{-}\right)+\gamma \widetilde{G}=\text { constant },
$$

whenever $V_{G}\left(\widetilde{G}, x, s_{-}\right)=-\gamma$.

Moreover, if we define $\operatorname{SP}\left(\gamma(G), x, s_{-}\right)=V\left(G, x, S_{-}\right)+\gamma(G) G$, we obtain

$$
\begin{aligned}
S P\left(\gamma, x, s_{-}\right)= & \max _{\left(a_{s}\right),\left(\pi_{s}\right)} \min _{\left(\lambda_{s}\right)} \sum_{s} \Pi\left(s_{-}, s\right) r\left(x_{s}, a_{s}, s\right) \\
& +\beta \pi_{s}\left(V^{L}\left(\gamma+\lambda_{s}, x_{s}^{\prime}\right)+\left(\gamma+\lambda_{s}\right) G^{L}\left(\gamma+\lambda_{s}, x_{s}^{\prime}\right)\right) \\
& +\beta\left(1-\pi_{s}\right)\left(V^{H}\left(\gamma+\lambda_{s}, x_{s}^{\prime}\right)+\left(\gamma+\lambda_{s}\right) G^{H}\left(\gamma+\lambda_{s}, x_{s}^{\prime}\right)\right) \\
& +\gamma g\left(x_{s}, a_{s}, s\right)+\lambda_{s}\left(g\left(x_{s}, a_{s}, s\right)-\bar{g}_{1}\right)
\end{aligned}
$$

subject to $x_{s}^{\prime}=\zeta\left(x_{s}, a_{s}, s\right), \quad p\left(x_{s}, a_{s}, s\right) \geqslant 0, \quad \forall s$.

But note that with Eq. (14) the optimal value of this problem can be derived from the optimal value of the social planning problem from the previous section. Simply note that

$$
S P\left(\gamma+\lambda_{s}, x, s\right)=V^{L}\left(\gamma+\lambda_{s}, x_{s}^{\prime}\right)+\left(\gamma+\lambda_{s}\right) G^{L}\left(\gamma+\lambda_{s}\right)=V^{H}\left(\gamma+\lambda_{s}, x_{s}^{\prime}\right)+\left(\gamma+\lambda_{s}\right) G^{H}\left(\gamma+\lambda_{s}, x_{s}^{\prime}\right) .
$$

By changing the timing in the problem of Section 3, it is now clear that we obtain the same value.

\section{Three simple examples}

Here we consider a simple partnership model in which there is a principal and an agent, and the principal must have the participation of the agent in order to run a project which produces a pie of size 1 every period that can be split between the two of them. Each period the principal gets to choose the amount of the pie he eats, $a$, and this implies the amount that the agent eats, $1-a$. The within period reward functions for the principal is $\log (a)$ and for the agent is $\log (1-a)$. Both are expected utility maximizers and both discount the future at rate $\beta$.

The agent has an initial outside opportunity, and then each period draws an outside opportunity. These opportunities put an initial ex ante lower bound on his payoff, which we call the participation constraint, and a conditional lower bound, which we call an incentive constraint. The ex ante outside opportunity which goes into the participation constraint is $\bar{g}_{2}=\frac{1}{1-\beta} \log (1 / 5)$. Assume that in every period there are two states of the world $s \in\{l, h\}$ which are i.i.d. and equiprobable. The conditional opportunity of the agent depends on the shock, $\bar{g}_{1}(l)=\frac{1}{1-\beta} \log (\epsilon)$ and $\bar{g}_{1}(h)=\frac{1}{1-\beta} \log (2 / 3)$. These opportunities determine the conditional incentive constraint.

We will assume that $a$ is bounded between $\varepsilon$ and $1-\varepsilon$, where $\varepsilon$ is a small number that serves to bound the payoffs of the agent and the principal. We will consider two cases: (i) the set of actions $a$ is convex and equal to $[\varepsilon, 1-\varepsilon]$, and (ii) the set of actions is not convex. In the nonconvex case, we will allow for public randomization in order to convexify the set of payoffs. If the set of possible actions $a$ by the principal is convex, then one can show that the Pareto frontier is strictly concave, however if the action set is not convex, then one can easily construct examples in which the Pareto frontier is weakly concave but not strictly so. 


\subsection{Example 1: No flat spots}

Assume that the set of possible actions are $a \in[\epsilon, 1-\epsilon]$ for some small $\epsilon>0$. We start by considering a recursion using promised utility and characterize the optimal actions. Then we consider a recursion using the cumulative multiplier and show that it generates the same optimal actions.

To begin, we let $V(G)$ denote the ex ante Pareto frontier, then the Lagrangian for this problem can be written as

$$
\begin{aligned}
L(G)= & \max _{[\psi(a, s), G(s, a)][\gamma, \lambda(s, a), v(a, s), \varphi(s)]} E\left\{\int_{a}[\log (a)+\beta V(G(s, a))] d \psi(a, s)\right\} \\
& +\gamma E\left\{\int_{a}[\log (1-a)+\beta G(s, a)] d \psi(a, s)-G\right\} \\
& +\sum_{s} \int_{a} \lambda(s, a)\left[\log (1-a)+\beta G(s, a)-\bar{g}_{1}(s)\right] d \psi(a, s) \\
& +E\left\{\int_{a} \psi(a, s) v(a, s) d a\right\}+E\left\{\varphi(s)\left[1-\int_{a} \psi(a, s) d a\right]\right\} .
\end{aligned}
$$

We have added constraints to ensure the probabilities $\psi(a, s)$ are nonnegative and sum to one; $v(a, s)$ and $\varphi(s)$ are multipliers on these constraints. In addition, the incentive constraint is multiplied by the p.d.f. over the action set to ensure that it holds for all actions taken with positive probability. The solution to this problem implicitly determines the Pareto frontier since $V(G)=L(G)$. And, when we set $G=\bar{g}_{2}$, the value of the PC, we generate the solution to our contracting problem.

This problem leads to the following f.o.c.s with respect to the probabilities

$$
\begin{aligned}
& \log (a(h))+\beta V(G(h, a))+(\gamma+\lambda(h, a))[\log (1-a(h))+\beta G(h, a)]-\lambda(h, a) \bar{g}_{1}(s)+v(a, h)-\varphi(h)=0, \\
& \log (a(l))+\beta V(G(l, a))+\gamma[\log (1-a(l))+\beta G(l, a)]+v(a, l)-\varphi(l)=0,
\end{aligned}
$$

and the following f.o.c.s with respect to continuation payoffs

$$
V^{\prime}(G(h, a))+\gamma+\lambda(h, a)=0,
$$

and

$$
V^{\prime}(G(l, a))+\gamma=0 .
$$

When the incentive constraint does not bind, as it cannot in state $l$, the f.o.c. for the continuation payoff uniquely determines it as the solution to

$$
V^{\prime}(G)=\gamma .
$$

Given this, the f.o.c. w.r.t. the probability implies that

$$
a \in \arg \max _{a}\{\log (a)+\gamma \log (1-a)\},
$$

which, since the set of possible $a$ 's is convex, is characterized by the first-order condition

$$
\frac{1}{a(l)}=\gamma \frac{1}{1-a(l)} \text {. }
$$

When the incentive constraint binds, as it can in state $h$, we get that

$$
G(a)=\beta^{-1}\left[\log (1-a)-\bar{g}_{1}(h)\right],
$$

and using our f.o.c.s for $a(h)$ we get that the optimal choice is a solution to

$$
a \in \arg \max _{a}\left\{\log (a)+\beta V\left(\beta^{-1}\left[\log (1-a)-\bar{g}_{1}(h)\right]\right)\right\},
$$

and hence that

$$
\frac{1}{a}+V^{\prime}\left(\beta^{-1}\left[\log (1-a)-\bar{g}_{1}(h)\right]\right) \frac{1}{1-a}=0 .
$$

This condition can be rewritten in terms of our multipliers as 


$$
\frac{1}{a(h)}=(\gamma+\lambda(h, a)) \frac{1}{1-a(h)} .
$$

The solution here is unique when the choice set for $a$ is convex, and conditions (17)-(21) determine the solution given the multipliers $\gamma$ and $\lambda(h)$, and the fact that $\lambda(l)=0$.

Since the continuation utility is $G(a, s)$ next period, it follows that the multiplier on the promised utility condition in the update problem will be $\gamma+\lambda(h, a)$. Continuing in this manner, we can construct the future sequence of multiplier values. If we denote the multiplier on the PC by $\gamma_{0}$ and the multipliers on the IC constraint in history state $\left(h^{t-1}, h\right)$ and $\left(h^{t-1}, l\right)$ by $\beta^{t} \lambda\left(h^{t}\right) \operatorname{Pr}\left(h^{t}\right)$, we get the first-order condition for $a\left(h^{t}\right)$,

$$
\frac{1}{a\left(h^{t}\right)}-\left[\gamma_{0}+\sum_{h^{j} \preccurlyeq h^{t}} \lambda\left(h^{j}\right)\right] \frac{1}{1-a\left(h^{t}\right)}=0,
$$

where "ß" means predecessor history and includes the current history. The fact that the IC multipliers for each state along an event tree are additive motivated Marcet and Marimon to focus on the recursive multiplier

$$
\gamma\left(h^{t-1}\right)=\gamma_{0}+\sum_{h^{j} \preccurlyeq h^{t-1}} \lambda\left(h^{j}\right),
$$

in which case the multiplier in $h^{t}$ becomes $\gamma\left(h^{t-1}\right)+\lambda\left(h^{t}\right)$ in the above expression. Since this alternative representation is equivalent to the original representation, and since that representation was necessary and sufficient, it follows that this representation is as well.

If we go a step further and consider a recursive planning problem in which the state variable for the agent is his recursive multiplier $\gamma$, we get Marcet and Marimon's formulation

$$
F(\gamma, s)=\max _{a \in A} \min _{\lambda \geqslant 0} \log (a)+\gamma \log (1-a)+\lambda\left[\log (1-a)-\bar{g}_{1}(s)\right]+\beta E_{s} F(\gamma+\lambda, \tilde{s}),
$$

where for notational simplicity we have consider only deterministic action choices. The first-order conditions with respect to $a$ is simply

$$
\frac{1}{a}-\gamma \frac{1}{1-a}-\lambda \frac{1}{1-a}=0
$$

and hence, just as in the promised utility approach, $a=\frac{1}{1+\gamma+\lambda}$ if the resulting $a \in[\epsilon, 1-\epsilon]$, which will always be the case.

The first-order condition with respect to $\lambda$ is

$$
\log (1-a)-\bar{g}_{1}(s)+\beta E_{s} \frac{d}{d \lambda} F(\gamma+\lambda, \tilde{s})=0 .
$$

From the envelope condition

$$
\frac{d}{d \gamma} F(\gamma, s)=\log (1-a)-\bar{g}_{1}(s)+\beta E_{s} \frac{d}{d \gamma} F(\gamma+\lambda, \tilde{s}),
$$

and hence by recursively substituting we obtain that

$$
E_{s} \frac{d}{d \lambda} F(\gamma+\lambda, \tilde{s})=G(s)
$$

i.e. exactly the continuation payoff that we obtained under the utility promise approach. Since the action $a$ is uniquely determined by the multipliers, it follows that the continuation payoff is also uniquely determined. Moreover, when we substitute this back into our original first-order condition for $\lambda$ we obtain the IC constraint, which ensures that it is satisfied as an equality whenever $\lambda>0$.

Given that we have determined the optimal action and implicitly the optimal continuation payoff as a function of the multipliers $\gamma+\lambda$, we only need to solve for $\lambda(\gamma, s)$ and as well as the period zero $\gamma_{0}$ which is needed to satisfy the participation constraint.

To completely specify $F$, first define the function

$$
a(\gamma)=1 /(1+\gamma)
$$

which solves (20). Then note that since $1-a(\gamma) \geqslant 2 / 3$ for all $\gamma \geqslant 2$, it follows that the IC constraint cannot bind in this case and hence that for all $\gamma \geqslant 2$

$$
F_{l}(\gamma)=F_{h}(\gamma)=\frac{1}{1-\beta}(\log (a(\gamma))+\gamma \log (1-a(\gamma))),
$$


and $\lambda(\gamma)=0$. For any $\gamma<2$ and $s=h$, the IC constraint binds and it follows that $\lambda(\gamma)=2-\gamma, a=a(\gamma+\lambda)$ from $(21)$, and hence that

$$
F_{h}(\gamma)=\frac{1}{1-\beta}[\log (a(2))+2 \log (1-a(2))]-(2-\gamma) \bar{g}_{1}(h) .
$$

For $\gamma<2$ and $s=l$ the IC constraint will not bind today and hence $\lambda=0$. But with probability $1 / 2$ it will bind tomorrow in state $h$. This implies that

$$
\begin{aligned}
F_{l}(\gamma) & =\log (a(\gamma))+\gamma \log (1-a(\gamma))+\frac{\beta}{2}\left[F_{l}(\gamma)+F_{h}(\gamma)\right] \\
& =\beta^{A}[\log (a(\gamma))+\gamma \log (1-a(\gamma))]+\beta^{B}[\log (a(2))+2 \log (1-a(2))],
\end{aligned}
$$

where

$$
\beta^{A}=\left[1+0.5 \beta+(0.5 \beta)^{2}+\cdots\right] \text { and } \beta^{B}=(1-\beta)^{-1}-\beta^{A} .
$$

Given these results, we can also express the payoffs to the principal and the agent in terms of the cumulative multiplier. In state $s=l$ the payoffs for the principal and the agent respectively are

$$
V_{l}(\gamma)= \begin{cases}\log (a(\gamma)) \beta^{A}+\frac{\log (a(2))}{1-\beta} \beta^{B} & \text { if } \gamma \leqslant 2, \\ \log (a(\gamma))(1-\beta)^{-1} & \text { o.w. }\end{cases}
$$

and

$$
G_{l}(\gamma)= \begin{cases}\log (1-a(\gamma)) \beta^{A}+\bar{g}_{1}(h) \beta^{B} & \text { if } \gamma \leqslant 2, \\ \log (1-a(\gamma))(1-\beta)^{-1} & \text { o.w. }\end{cases}
$$

In state $s=h$ the payoffs are

$$
V_{h}(\gamma)= \begin{cases}\log (a(2))(1-\beta)^{-1} & \text { if } \gamma \leqslant 2 \\ \log (a(\gamma))(1-\beta)^{-1} & \text { o.w. }\end{cases}
$$

and

$$
G_{h}(\gamma)= \begin{cases}\bar{g}_{1}(h) & \text { if } \gamma \leqslant 2, \\ \log (1-a(\gamma))(1-\beta)^{-1} & \text { o.w. }\end{cases}
$$

We plot the Pareto frontier implied by these payoffs when we set $\beta=0.95$ and $\epsilon=0.1$ for state $s=l$ in the first panel of Fig. 1 and the payoff function $G_{l}(\gamma)$ for the agent in the second panel.

\subsection{Example 2: Flat spots}

We now change the previous example and assume that $a \in\{\epsilon\} \cup[0.5,1-\epsilon]$. The previous argument does not apply since the action set is not convex and there can potentially be multiple actions which satisfy the first-order conditions given the multipliers. In particular, at $\gamma=\gamma^{*}$ there are two solutions to (19) given the restricted action space, $a=\epsilon$ and $a=0.5$, where

$$
\gamma^{*}=\frac{\log (0.5)-\log (\epsilon)}{\log (1-\epsilon)-\log (0.5)}>1 .
$$

This creates a problem with respect to the first-order condition for $\lambda$, (22), in the recursive multiplier approach since the optimal action is not unique at $\gamma+\lambda=\gamma^{*}$. In addition, there is a flat spot in the Pareto frontier at $\gamma^{*}, F\left(\gamma^{*}, \tilde{s}\right)$ is not differentiable at this point and since the sub-differential is not unique, there are a continuum of continuation payoffs associated with $\gamma^{*}$. Because of this issue, the simple recursive multiplier approach cannot ensure that the previous periods' constraints will be satisfied. But we can keep track of the entire sub-differential through two extreme points. Moreover, by appropriately randomizing over these extreme points and subsequently restricting ourselves to the appropriate extreme point, we can overcome this difficulty.

For a $\gamma>\gamma^{*}$ the corner solution $a=\epsilon$ is optimal, the (IC) constraint does not bind, hence $\lambda=0$, and $F_{S}(\gamma)=$ $\frac{1}{1-\beta}(\log (\epsilon)+\gamma \log (1-\epsilon))$ for both $s=1,2$.

At $\gamma=\gamma^{*}$ the optimal solutions (to the recursive Pareto problem) are all randomizations over 0.5 and $\epsilon$, yielding 0.5 or $1-\epsilon$ to the agent. Since any $\gamma>\gamma^{*}$ implies the agent getting $1-\varepsilon$ forever, the IC constraints cannot possibly bind at $\gamma^{*}$ and $\lambda=0$. Hence, the payoff to the agent conditional on the current action is

$$
\log (a)+\frac{\beta}{1-\beta}[\pi \log (1-\epsilon)+(1-\pi) \log (0.5)]
$$



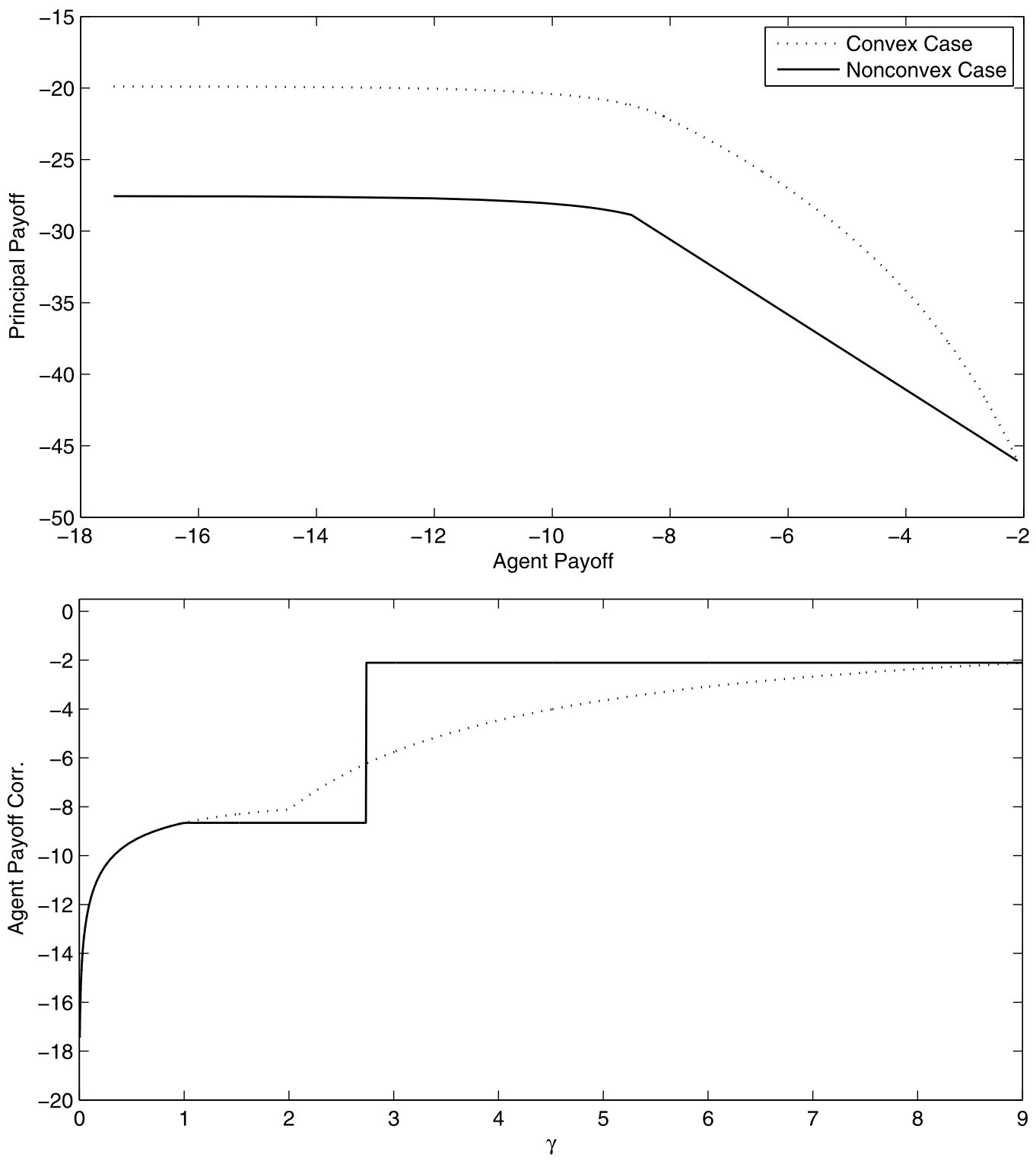

Fig. 1. First panel: Pareto frontiers. Second panel: Agent payoff correspondences.

for $a=0.5$ or $\epsilon$ and $\pi \in[0,1]$. To satisfy the IC in state $s=h$, we need that this payoff equals $\bar{g}_{1}(h)$, so clearly not any randomization will do.

If we let $G_{s}^{H}\left(\gamma^{*}\right)$ denote the high payoff to the agent in state $s$ and $G_{s}^{L}\left(\gamma^{*}\right)$ the lowest, then randomizing over extreme payoffs in the appropriate manner will allow us to achieve the efficient outcome. Clearly at $\gamma=\gamma^{*}$, the highest possible outcome for the agent is to set his consumption equal to $1-\epsilon$ forever, and this implies that $G_{s}^{H}\left(\gamma^{*}\right)=\frac{1}{1-\beta} \log (1-\epsilon)$, $s=l, h$, and that the ex ante high payoff $G^{H}\left(\gamma^{*}\right)$ is equal to this as well.

Next consider recursively defining $G_{s}^{L}\left(\gamma^{*}\right)$ as

$$
G_{s}^{L}\left(\gamma^{*}\right)=\min _{\pi \in[0,1], a \in\{0.5,1-\epsilon\}} \log (1-a)+\beta\left(\pi G^{H}\left(\gamma^{*}\right)+(1-\pi) G^{L}\left(\gamma^{*}\right)\right)
$$

subject to

$$
G_{s}^{L}\left(\gamma^{*}\right) \geqslant \bar{g}_{1}(s)
$$

and where

$$
G^{L}\left(\gamma^{*}\right)=0.5\left[G_{l}^{L}\left(\gamma^{*}\right)+G_{h}^{L}\left(\gamma^{*}\right)\right] .
$$


Clearly, we will want to make $\pi$ and $a$ as small as possible subject to the incentive constraint. Since the incentive constraint has to hold ex post, we cannot randomize over $a$ to satisfy this constraint. However, we can do so by randomizing over $G^{L}$ and $G^{H}$, which means that we are randomizing over actions tomorrow. Since the incentive constraint doesn't bind in the low state, it follows that $\pi=0, a=0.5$, and

$$
G_{l}^{L}\left(\gamma^{*}\right)=\log (0.5)+\frac{\beta}{2}\left[G_{l}^{L}\left(\gamma^{*}\right)+G_{h}^{L}\left(\gamma^{*}\right)\right]
$$

Since the incentive constraint binds in the high state $G_{h}^{L}\left(\gamma^{*}\right)=\bar{g}_{1}(h)$, Eq. (23) then yields directly

$$
G_{l}^{L}\left(\gamma^{*}\right)=\frac{1}{1-\beta / 2}\left(\log (0.5)+0.5 \beta \bar{g}_{1}(h)\right) .
$$

In terms of our formal notation we denote the policy here as $\omega_{l}^{L}\left(\gamma^{*}\right)=(a=0.5, \lambda=0, \pi=0)$.

The associated actions are clear in the low state - in the high state they depend on $\epsilon$ and the discount factor. If there exists a $\pi$ such that

$$
\log (0.5)+\beta\left(\pi\left(0.5 G_{h}^{H}+0.5 G_{l}^{H}\right)+(1-\pi)\left(0.5 G_{h}^{L}+0.5 G_{l}^{L}\right)\right)=\bar{g}_{1}(h),
$$

then the optimal action is 0.5 , otherwise it must be $\epsilon$. Note that there might be a region where the action is not unique and both $1 / 2$ and $\epsilon$ do the job with the right probabilities. Let the policy function $\omega_{h}^{L}\left(\gamma^{*}\right)$ denote the appropriate action $a$, $\lambda=0$, and the randomization $\pi$.

For any $\gamma<\gamma^{*}$ in shock $h$ the (IC) constraint binds, the optimal $\lambda\left(\gamma_{0}\right)=\gamma^{*}-\gamma_{0}$ and the associated policy is $\omega_{h}^{i}(h)$ which gives the action and randomization in $\omega_{h}^{L}\left(\gamma^{*}\right)$ and yields payoff $\bar{g}_{1}(h)$ for the agent.

For $\gamma<\gamma^{*}$ and shock $l$ things are a bit more complicated than in the convex case. Because there is a kink in the Pareto frontier at $a=0.5$ there is a range of values for the multiplier $\gamma$ that support the same action. Because the sub-differential is convex we only have to consider two cases when $\gamma_{0}<\gamma^{*}$ and the shock is $l$. If $\gamma_{0} \in\left(1, \gamma^{*}\right)$ then $a=0.5$. If $1 \geqslant \gamma_{0} \geqslant \frac{1}{1-\epsilon}-1$ the optimal $\lambda=0$ and the unique policy is determined by $a=\frac{1}{1+\gamma_{0}}$. The participation constraint pins down $\gamma_{0}$.

Implicit in our discussion is that for $\gamma<\gamma^{*}, G_{s}^{H}(\gamma)=G_{s}^{L}(\gamma)$. The reason for this is straightforward. At $h$, there is initially no randomization, the $\gamma+\lambda$ brings one to the flat spot but it is clear that the strategy is uniquely determined as the one that yields $\bar{g}_{1}(h)$. While at $s=l$, the IC constraint does not bind, $\lambda=0$, and the optimal action is uniquely determined.

Note that the randomization $\pi$ is identical for both shocks in the subsequent period. This is only possible because we randomize over the two extreme points of two intervals, describing the sets $\mathcal{G}\left(s^{\prime}\right)$ for the two possible shocks $s^{\prime}=1,2$ in the next period.

Given these results, we can express the payoffs to the principal and the agent in terms of the cumulative multiplier for the nonconvex case as well. Assume that there exists a randomization $\pi$ which is the solution to

$$
\log (0.5)+\beta \frac{\pi \log (1-\varepsilon)+(1-\pi) \log (0.5)}{1-\beta}=\bar{g}_{1}(h)
$$

(so we can use action 0.5 as opposed to $\epsilon$ ). Given this randomization, the payoffs for all $\gamma \neq \gamma^{*}$ are given by

$$
V_{l}^{j}(\gamma)= \begin{cases}\log (a(\gamma)) \beta^{A}+\frac{\log (a(2))}{1-\beta} \beta^{B} & \text { if } \gamma \leqslant 1, \\ \log (a(1)) \beta^{A}+\beta^{B} \frac{\pi \log (\varepsilon)+(1-\pi) \log (0.5)}{1-\beta} & \text { if } \gamma \in\left[1, \gamma^{*}\right), \\ \log (a(\gamma))(1-\beta)^{-1} & \text { if } \gamma>\gamma^{*}\end{cases}
$$

and by

$$
G_{l}^{j}(\gamma)= \begin{cases}\log (1-a(\gamma)) \beta^{A}+\bar{g}_{1}(h) \beta^{B} & \text { if } \gamma \leqslant 1, \\ \log (1-a(1)) \beta^{A}+\bar{g}_{1}(h) \beta^{B} & \text { if } \gamma \in\left[1, \gamma^{*}\right), \\ \log (1-a(\gamma))(1-\beta)^{-1} & \text { if } \gamma>\gamma^{*},\end{cases}
$$

for $j=H, L$. For all $\gamma<\gamma^{*}$ the state $h$ payoffs are very simple since the incentive constraint binds and hence $G_{h}^{j}(\gamma)=\bar{g}_{1}(h)$ which pins down $V_{h}^{j}(\gamma)$. For $\gamma>\gamma^{*}, G_{h}^{j}(\gamma)=G_{l}^{L}(\gamma)$ and $V_{h}^{j}(\gamma)=V_{l}^{L}(\gamma)$.

For $\gamma=\gamma^{*}$ we have to differentiate between the low and high payoffs, and

$$
\begin{aligned}
& G_{s}^{H}\left(\gamma^{*}\right)=\log (1-\varepsilon)(1-\beta)^{-1}, \\
& V_{s}^{H}\left(\gamma^{*}\right)=\log (\varepsilon)(1-\beta)^{-1},
\end{aligned}
$$

and

$$
\begin{aligned}
& G_{l}^{L}\left(\gamma^{*}\right)=G_{l}^{L}(1), \\
& V_{l}^{L}\left(\gamma^{*}\right)=V_{l}^{L}\left(\gamma^{*}\right) .
\end{aligned}
$$


In Fig. 1, we also plot the Pareto frontier and the payoff correspondence for the agent when $s=l$ in the nonconvex case under the same parameterization as we did for the convex case. In the first panel plot of the Pareto frontiers we can clearly see how the Pareto frontier in the nonconvex is reduced due to utility loss from having to randomize relative to the convex case for all $\gamma \leqslant \gamma^{*}$. We can also see the linear segment with slope $-\gamma^{*}$ in the nonconvex case at $\gamma=\gamma^{*}$. With respect to the payoff correspondence plotted in the second panel, note that the correspondence is degenerate for all $\gamma \neq \gamma^{*}$, and hence $G_{l}^{H}(\gamma)=G_{l}^{L}(\gamma)$, while at $\gamma=\gamma^{*}$ these are the respective maximum and minimum payoffs for the correspondence.

\subsection{Example 3: How do flat spots propagate}

We now change the example by assuming that the action space can stochastically switch from the continuous action space $(c)$ to the discrete action space $(d)$ and stay there forever. The state space is now $s \in\{(l, c),(h, c),(l, d),(h, d)\}$, where the draws of $l$ and $h$ continue to be i.i.d. and equi-probable, and the probability of switching from $c$ to $d$ next period is $\rho$, while $d$ is an absorbing state. If the state is $(i, c)$ for $=l, h$ then the action space is $A(i, c)=[\epsilon, 1-\epsilon]$, while if it is $(i, d)$ it is $A(i, d)=\{\epsilon\} \cup[0.5,1-\epsilon]$. We assume that the initial states are either $(l, c)$ or $(l, d)$.

The recursive planning problem is

$$
F(\gamma, s)=\max _{a \in A(s)} \min _{\lambda \geqslant 0} \log (a)+\gamma \log (1-a)+\lambda\left[\log (1-a)-\bar{g}_{1}(s)\right]+\beta E_{s} F(\gamma+\lambda, \tilde{s}),
$$

and the first-order conditions are still

$$
\frac{1}{a}-\gamma \frac{1}{1-a}-\lambda \frac{1}{1-a}=0
$$

with respect to $a$ and

$$
\log (1-a)-\bar{g}_{1}(s)+\beta E_{s} F^{\prime}=0
$$

with respect to $\lambda$, which implies that $a=\frac{1}{1+\gamma+\lambda}$, if the action space is $A(i, c)$ and the resulting $a \in[\epsilon, 1-\epsilon]$.

Clearly, when the action space is $A(i, d)$, the solution to this problem will work just like in Example 2, and there will be a flat spot on the conditional Pareto frontier at slope $\gamma^{*}$. For this reason, continue to let $G_{l}^{j}$ for $j=H$ and $L$ denote the high and low payoffs respectively in the discrete case when $s=l$. In state $(l, c)$, the payoff to the agent, $W_{l}(\gamma)$, will be implicitly given by

$$
\begin{aligned}
W_{l}(\gamma)= & \log (1-a(\gamma+\lambda))+\frac{\beta}{2}(1-\rho) W_{l}(\gamma+\lambda)+\rho \frac{\beta}{2}\left[\left(\pi G_{l}^{H}(\gamma+\lambda)+(1-\pi) G_{l}^{L}(\gamma+\lambda)\right)\right] \\
& +\frac{\beta}{2}[(1-\rho)+\rho] \bar{g}_{1}(h),
\end{aligned}
$$

where $\lambda$ is chosen to satisfy the incentive constraint that this payoff must be at least $\bar{g}_{1}(l)$. If $\lambda>0$ then this payoff must be $\bar{g}_{1}(l)$, and hence is uniquely defined. If $\gamma \neq \gamma^{*}$ and $\lambda=0$, then $G_{l}^{H}(\gamma)=G_{l}^{L}(\gamma)$ and the payoff is again uniquely defined. However if $\gamma=\gamma^{*}$ and $\lambda=0$, then $G_{l}^{H}(\gamma)>G_{l}^{L}(\gamma)$ and the ability to randomize over this two continuation payoffs will induce a flat section on the Pareto frontier today.

Similarly, in state $(h, c)$ the payoff to the agent, $W_{h}(\gamma)$, will be implicitly given by

$$
\begin{aligned}
W_{h}(\gamma)= & \log (1-a(\gamma+\lambda))+\frac{\beta}{2}(1-\rho) W_{h}(\gamma+\lambda)+\rho \frac{\beta}{2}\left[\left(\pi G_{l}^{H}(\gamma+\lambda)+(1-\pi) G_{l}^{L}(\gamma+\lambda)\right)\right] \\
& +\frac{\beta}{2}[(1-\rho)+\rho] \bar{g}_{1}(h),
\end{aligned}
$$

where $\lambda$ is chosen to satisfy the incentive constraint that this payoff must be at least $\bar{g}_{1}(h)$. If with $\gamma=\gamma^{*}, \lambda=0$ and $\pi=1$ the payoff $W_{h}(\gamma)>\bar{g}_{1}(h)$, then here too the payoff to the agent will be indeterminate when $\gamma=\gamma^{*}$.

This example illustrates that flat sections in the continuation Pareto frontier do induce flat spots in the current Pareto frontier, but only at the specific value of the multiplier. ${ }^{8}$

\section{Computational considerations}

We show in this section that the insights from the simple example above carry over to more complicated models. For simplicity we assume that there is no physical state and that there is only one agent and a principal. For models with a physical state variable (such as for example capital), many of the insights of this section will still hold true as long as one makes strong enough assumptions on the law of motion, $\zeta$. For models with several agents, the state space becomes highdimensional and even standard numerical dynamic programming is challenging. Cai and Judd (2010) show how to handle

8 This result is sensitive to the assumption that the principal and the agent discount at the same rate. 
several continuous state variables for the standard case but it is beyond the scope of this paper to extend their methods to recursive contracts.

Here, we propose a simple value function iteration to solve for the optimal policy even when there are flat spots in the Pareto frontier. In the case without physical state, the recursive planner problem simplifies to

$$
F(\gamma, s)=\max _{\psi \in \Psi} \min _{\lambda \geqslant 0} \sum_{a \in \operatorname{supp}(\psi)} \psi(a)\left[r(a, s)+\gamma g(a, s)+\lambda_{a}\left(g(a, s)-\bar{g}_{1 s}\right)+\beta E_{s} F\left(\gamma+\lambda_{a}, s^{\prime}\right)\right]
$$

subject to

$$
p(a, s) \geqslant 0, \quad \forall a \in \operatorname{supp}(\psi) .
$$

We assume that the set of available actions $\mathcal{A} \subset \mathbb{R}^{n}$ can be written as a finite union of compact and convex sets. To simplify notation, we assume that this is described by the constraint $p(a, s) \geqslant 0$ where for each $s, p(., s): \mathbb{R}^{n} \rightarrow \mathbb{R}^{m}$. In the example above, $\mathcal{A}$ was one-dimensional and consisted of the union of intervals, which obviously can be described by inequalities. We assume that reward functions are differentiable on $\mathcal{A}$. We assume that $r(., s), g(., s)$ and $p(., s)$ are twice continuously differentiable for each shock $s$. Finally we assume that for each $s$, the reward function $r(., s)$ is monotone in the actions - this is a strong assumption that can be relaxed, but it simplifies the analysis.

In principle, the proof of Theorem 1 gives rise directly to a simple algorithm to compute $F$. Given an admissible range for $\gamma,[0, \bar{\gamma}]$ we start with functions $F_{0}:[0, \bar{\gamma}] \times \mathcal{S} \rightarrow \mathbb{R}$ that are smooth and convex in $\gamma$. We then iterate the following recursion until some natural stopping criterion is satisfied:

$$
F_{n}(\gamma, s)=\max _{\psi \in \Psi} \min _{\bar{\gamma}-\gamma \geqslant \lambda \geqslant 0} \sum_{a \in \operatorname{supp}(\psi)} \psi(a)\left[r(a, s)+\gamma g(a, s)+\lambda_{a}\left(g(a, s)-\bar{g}_{1 s}\right)+\beta E_{s} F_{n-1}\left(\gamma+\lambda_{a}, s^{\prime}\right)\right]
$$

subject to

$$
p(a, s) \geqslant 0, \quad \forall a \in \operatorname{supp}(\psi) .
$$

The computational challenge is to solve this step numerically and to construct an approximation for $F_{n}$ given an approximation for $F_{n-1}$.

\subsection{Some preliminary results}

The following lemma is standard, but important to point out.

Lemma 4. Suppose $F_{n-1}(., s)$ is convex and continuous for all shocks $s$. Then the function $F_{n}(., s)$ defined by (25) is continuous and convex for all $s \in \mathcal{S}$.

Proof. Continuity of $F(., s)$ is standard. To show that it is also convex we need to verify that for any $s \in \mathcal{S}, \gamma, \tilde{\gamma} \geqslant 0$ and $\mu \in$ $[0,1], F_{n}(\mu \gamma+(1-\mu) \tilde{\gamma}, s) \leqslant \mu F_{n}(\gamma, s)+(1-\mu) F_{n}(\tilde{\gamma}, s)$. Note that if $\left(\psi^{*}, \lambda^{*}\right)$ solves the problem for $\gamma^{*}=\mu \gamma+(1-\mu) \tilde{\gamma}$, the action $\psi^{*}$ is also feasible for both $\gamma$ and $\tilde{\gamma}$. Furthermore, note that if $F_{n-1}$ is convex, for any $a^{*}$ in the support of $\psi^{*}$ we must have

$$
\begin{aligned}
& \mu\left[\min _{\lambda \geqslant 0} r\left(a^{*}, s\right)+\gamma g\left(a^{*}, s\right)+\lambda\left(g\left(a^{*}, s\right)-\bar{g}_{1 s}\right)+\beta E_{s} F_{n-1}\left(\gamma+\lambda, s^{\prime}\right)\right] \\
& \quad+(1-\mu)\left[\min _{\lambda \geqslant 0} r\left(a^{*}, s\right)+\tilde{\gamma} g\left(a^{*}, s\right)+\lambda\left(g\left(a^{*}, s\right)-\bar{g}_{1 s}\right)+\beta E_{s} F_{n-1}\left(\tilde{\gamma}+\lambda, s^{\prime}\right)\right] \\
& \geqslant \min _{\lambda \geqslant 0} r\left(a^{*}, s\right)+\gamma^{*} g\left(a^{*}, s\right)+\lambda\left(g\left(a^{*}, s\right)-\bar{g}_{1 s}\right)+\beta E_{s} F_{n-1}\left(\gamma^{*}+\lambda, s^{\prime}\right) .
\end{aligned}
$$

Therefore, we must have that

$$
\begin{aligned}
& \mu F(\gamma, s)+(1-\mu) F(\tilde{\gamma}, s) \\
& \quad \geqslant \min _{\lambda \geqslant 0} r\left(a^{*}, s\right)+\gamma^{*} g\left(a^{*}, s\right)+\lambda\left(g\left(a^{*}, s\right)-\bar{g}_{1 s}\right)+\beta E_{s} F_{n-1}\left(\gamma^{*}+\lambda, s^{\prime}\right) \\
& \quad=F(\mu \gamma+(1-\mu) \tilde{\gamma}, s) .
\end{aligned}
$$

This proves the lemma.

Continuous and convex functions are differentiable almost everywhere. Since we restrict attention to a finite domain for $\gamma$, there are at most finitely many points where $F_{n}$ is not differentiable. We refer to these as 'critical points'. For these points, we need to introduce the so-called sub-differential (see Rockafellar, 1970, for details). Let $\nabla F_{n}(\gamma, s)$ denote the sub-differential of $F_{n}$ with respect to $\gamma$. If $F_{n}$ is differentiable at $\gamma$, the map is single-valued and equal to the gradient 
of $F_{n}$. The points where $F_{n}$ fails to be differentiable are precisely those that correspond to a flat spot in the Pareto frontier. We can write $\nabla F_{n}(\gamma, s)=\left[F_{n-}^{\prime}(\gamma, s), F_{n+}^{\prime}(\gamma, s)\right]$, where $F_{n-}^{\prime}$ and $F_{n+}^{\prime}$ are the one-sided directional derivatives at $\gamma$. It is standard to see that given an action $a$, an interior solution for $\lambda$ must satisfy the first-order condition

$$
g(a, s)-\bar{g}_{1 s}+\beta E_{s} \partial\left(s^{\prime}\right)=0,
$$

with $\partial\left(s^{\prime}\right) \in \nabla F_{n}\left(\gamma+\lambda, s^{\prime}\right)$ for all $s^{\prime}$.

It is clear that for a given $(\gamma, s)$ and a given $\lambda$ the set of optimal actions (that are associated with that $\lambda$ ) can be determined without knowledge of $F$. If the problem is smooth it is simply a subset of the set of zeros of the equations

$$
D_{a} r(a, s)+(\gamma+\lambda) D g(a, s)+\eta D p(a, s)=0, \quad \eta_{i} p_{i}(a, s)=0,
$$

that satisfy $p(a, s) \geqslant 0$.

The randomization is then over all solutions to this system that yield the same value of the objective function.

Note that by the first-order conditions (26) for a given $\lambda$, if one of the reward functions is strictly monotone in the action, there can only be more than one solution if $\lambda=0$ or if we are at a point where $F_{n}\left(\gamma+\lambda, s^{\prime}\right)$ is not differentiable for some $s^{\prime}$.

Also note that if $a(\gamma+\lambda)$ and $a(\gamma+\tilde{\lambda})$ solve Eq. (27) for $\lambda$ and $\tilde{\lambda}$ respectively, and if $\lambda<\tilde{\lambda}$, we must have $g(a(\gamma+\lambda), s)<$ $g(a(\gamma+\tilde{\lambda}), s)$. Since $\nabla F$ is monotone, the first-order condition (26) then implies directly that there cannot be two different $(a, \lambda)$ and $(\tilde{a}, \tilde{\lambda})$ with $\tilde{\lambda} \neq \lambda$ that both solve the Bellman equation $(25)$.

\subsection{Computational details}

The main computational challenges is to identify those $\bar{\xi}=\bar{\gamma}+\bar{\lambda}$ points where there are several solutions to the maximization problem

$$
\max _{a} r(a, s)+\bar{\xi} g(a, s) \quad \text { s.t. } \quad p(a, s) \geqslant 0 .
$$

In some cases, as in the example above, these points might be obvious or can be solved for easily. In other cases, it might be impossible to solve for these points - in which case our method is not applicable (but in which case it seems hopeless to ever solve the problem at all). We focus on the intermediate case where methods from computational algebraic geometry can be used to find all solutions to a system of non-linear equations. We therefore assume now that $r(., s), g(., s)$ and $p(., s)$ are algebraic functions, i.e. functions that can be characterized by polynomial equations (such as rational functions, roots, etc). In this case one can go about the problem as follows. The system of first-order conditions (27) can be written as a series of polynomial equations. Given $\lambda+\gamma$, these equations have finitely many solutions which can all be obtained numerically. One convenient way to obtain these solutions is to use the so-called Gröbner bases (see Kubler and Schmedders, 2010). The basic idea behind this method is that given a polynomial system of equations with finitely many solutions Gröbner bases form an equivalent system that has a triangular form. One can solve for all solutions in the $n$-th variable by solving a univariate polynomial (for which there are efficient and reliable numerical methods), the solutions in all other variables are then simply polynomial functions in the $n$-th variable. Solving for all solutions can then be done with arbitrary precision. Routines to solve for Gröbner bases are available in most computer algebra system (such as Maple or Mathematica). In the Gröbner basis for our system, the variable $\xi=\lambda+\gamma$ can be treated as a parameter. Having a (polynomial) representation for all solutions to the first-order conditions as a function of $\xi$, one then needs to ask for which values of the parameter, $\xi$, two solutions to the first-order conditions give the same value of the objective function $r(a, s)+\bar{\xi} g(a, s)$. This problem can itself be formulated as a polynomial system and solved with Gröbner basis. As a result one obtains finitely many $\xi$ for which there are two solutions to the first-order conditions which give rise to the same value of the objective function. Finally one has to verify for each of these points that two values that solve the first-order conditions and give the same value of the objective functions are in fact global optimums, i.e. there is no other solution to the first-order conditions that gives a higher value. At the end, one obtains a finite number of 'critical' values for $\xi$ which we collect in the set $\Xi \subset[0, \bar{\gamma}]$.

After this initial step, the computation is more or less standard. We start with an initial guess of a single-valued policy and differentiable and convex value function. As in standard value function iteration (see e.g. Judd, 1998), we iterate backwards, solving the Bellman equation (25) at a finite number of predetermined points in each iteration and approximating $F_{n}$ by piecewise smooth functions, for example by splines.

For each $s \in \mathcal{S}$ the value function from the $n$-th iteration is given by $F_{n}(, s)$. Suppose there are $N_{n}$ points where the function is non-differentiable and that these critical points, $\Gamma_{n}=\left\{\gamma_{n}(1), \ldots, \gamma_{n}\left(N_{n}\right)\right\}$, are given as well are the left- and right-sided derivatives at these points, $F_{-}^{\prime}\left(\gamma_{n}(i)\right), F_{+}^{\prime}\left(\gamma_{n}(i)\right)$. Note that eventually these points will most likely include $\Xi$ but the set might be strictly larger because flat spots might propagate. Suppose also, we are given the value functions $G_{n}^{L}$ and $G_{n}^{H}$ for the principal and that we are given the set $\Xi$. For each $\gamma \in \Xi$ we need to first verify if there is a solution with $\lambda=0$ - in this case, we are at a critical point and $\gamma \in \Gamma_{n+1}$. Furthermore, we need to verify if for $\gamma \notin \Xi$, there is a solution with $\lambda>0$ so that $\gamma+\lambda \in \Gamma_{n}$. Given approximations for $F_{-}^{\prime}\left(\gamma_{n}(i)\right), F_{+}^{\prime}\left(\gamma_{n}(i)\right)$, this step simply reduces to checking an inequality. In our above example, we referred to this case as propagation of flat spots. In this case $\gamma$ itself becomes a critical point for iteration $n+1$, i.e. $\gamma \in \Gamma_{n+1}$. In all other cases $\gamma$ is not critical and there exists a unique solution to the 
Bellman equation, and $G_{n+1}^{L}=G_{n+1}^{H}$. For $\gamma \in \Gamma_{n+1}$ we can determine the set of optimal actions, $A(\gamma, s)$, using Gröbner basis and considering the system (27) above. We finally determine $G_{n+1}^{L}$ and $G_{n+1}^{H}$ as follows:

$$
\begin{aligned}
& G_{n+1}^{L}(\gamma, s)=\min _{(\underline{a}, \underline{\lambda}) \in A(\gamma, x, s), \pi \in[0,1]} g(\underline{a}, s)+\beta E_{s}\left(\pi G_{n}^{L}\left(\gamma+\underline{\lambda}, s^{\prime}\right)+(1-\pi) G_{n}^{H}\left(\gamma+\underline{\lambda}, s^{\prime}\right)\right), \\
& G_{n+1}^{H}(\gamma, s)=\max _{(\bar{a}, \bar{\lambda}) \in A(\gamma, s), \pi \in[0,1]} g(\bar{a}, s)+\beta E_{s}\left(\pi G_{n}^{L}\left(\gamma+\bar{\lambda}, s^{\prime}\right)+(1-\pi) G_{n}^{H}\left(\gamma+\bar{\lambda}, s^{\prime}\right)\right)
\end{aligned}
$$

subject to

$$
G^{H}(\gamma, s) \geqslant \bar{g}_{1}, \quad G^{L}(\gamma, s) \geqslant \bar{g}_{1}
$$

This finishes one iteration step and we iterate until successive $F_{n}$ and $F_{n-1}$ are 'close', i.e. the sup norm between these functions lies below some pre-specified stopping criterion.

For the case of one principal and one agent our computational method is really only a small extension of the standard Marcet-Marimon method. Instead of only keeping track of the multiplier and a mapping from the multiplier and the state to payoff from the social planning problem, $F(\gamma, s)$, here we also keep track of a mapping from the multiplier and the state to highest and lowest payoffs of the agent, $G^{H}(\gamma, s)$ and $G^{L}(\gamma, s)$, and which of these payoffs are to be used.

\section{Conclusion}

The recursive multiplier approach in Marcet and Marimon (1994) can be extended to models with flat spots in the Pareto frontier. In order for our method to be computationally tractable, the flat spots must be polytopes. This is obviously always the case if there is only one agent in addition to the principal. In the case of several agents, this will naturally be the case if the flat spots are generated lotteries over a finite number of actions. On the other hand, it is clearly possible to construct problems where the flat spot is a general convex set. For problems like this, neither the promised utility approach nor our method are likely to be feasible.

\section{References}

Aiyagari, R., Marcet, A., Sargent, T., Seppala, J., 2002. Journal of Political Economy 110 (6), 1220-1254.

Atkeson, A., Cole, H., 2005. A dynamic theory of executive compensation and optimal capital structure. NBER working paper \#11083.

Attanasio, O., Rios-Rull, J., 2000. Consumption smoothing in island economies: Can public insurance reduce welfare? European Economic Review 44 (7), $1225-1258$

Cai, Y., Judd, K.L., 2010. Stable and efficient computational methods for dynamic programming. Journal of the European Economic Association 8, 626-634.

Chien, Y., Cole, H., Lustig, H., 2011. A multiplier approach to understanding the macro implications of household finance. Review of Economic Studies 78 (1), 199-234.

Cole, H., Prescott, E., 1997. Valuation equilibrium with clubs. Journal of Economic Theory 74, 19-39.

Cooley, T., Marimon, R., Quadrini, V., 2004. Aggregate consequences of limited contract enforceability. Journal of Political Economy 112 (4), 817-847.

Dechert, W.D., 1982. Lagrange multiplier in infinite horizon discrete time optimal control models. Journal of Mathematical Economics 9, $285-302$.

Fukuda, K., 2004. From the zonotope construction to the Minkowski sum of convex polytopes. Journal of Symbolic Computation 38, $1261-1272$.

Judd, K., 1998. Numerical Methods in Economics. MIT Press, Cambridge.

Kehoe, P., Perri, F., 2002. International business cycles with endogenous incomplete markets. Econometrica 70 (3), $907-928$.

Khan, A., King, R., Wolman, A., 2003. Optimal monetary policy. Review of Economic Studies 70 (4), $825-860$.

Kubler, F., Schmedders, K., 2010. Tackling multiplicity of equilibria with Gröbner bases. Operations Research 58, $1037-1050$.

Luenberger, D.G., 1969. Optimization by Vector Space Methods. Wiley.

Marcet, A., Marimon, R., 1992. Communication, commitment, and growth. Journal of Economic Theory 58, $219-249$.

Marcet, A., Marimon, R., 1994. Recursive contracts. Discussion paper, Universitat Pompeu Fabra.

Marcet, A., Marimon, R., 2011. Recursive contracts. Economics working papers, ECO2011/15, European University Institute.

Messner, M., Pavoni, N., 2004. On the recursive saddle point method. IGIER working paper 255, Universita Bocconi.

Messner, M., Pavoni, N., Sleet, C., 2011. Recursive methods for incentive problems. Research memo.

Prescott, E., Townsend, R., 1984a. General competitive analysis in an economy with private information. International Economic Review 25 (1), 1-20.

Prescott, E., Townsend, R., 1984b. Pareto optima and competitive equilibria with adverse selection and moral hazard. Econometrica 52 (1), 21-46.

Rockafellar, R.T., 1970. Convex Analysis. Princeton University Press.

Rogerson, R., 1988. Indivisible labor, lotteries and equilibrium. Journal of Monetary Economics 21, 3-16.

Rustichini, A., 1998. Lagrange multiplier in incentive-constrained problems. Journal of Mathematical Economics 29, 365-380.

Spear, S., Srivastava, S., 1987. On repeated moral hazard with discounting. Review of Economic Studies 54, $599-617$.

Stokey, N., Lucas, R., 1989. Recursive Methods in Economic Dynamics. Harvard University Press. With E. Prescott. 